\title{
Airborne Wireless Sensor Networks for Airplane Monitoring System
}

\author{
Shang Gao $\mathbb{D}^{1},{ }^{1}$ Xuewu Dai, ${ }^{2}$ Yu Hang, ${ }^{1}$ Yuyan Guo, ${ }^{1}$ and Qian Ji ${ }^{1}$ \\ ${ }^{1}$ School of Mechanical Engineering, Nanjing University of Science and Technology, XiaoLingWei Street No. 200, Nanjing 210094, China \\ ${ }^{2}$ Faculty of Engineering and Environment, Northumbria University, Newcastle upon Tyne NE1 8ST, UK \\ Correspondence should be addressed to Shang Gao; shang.gao@njust.edu.cn
}

Received 11 January 2018; Revised 23 March 2018; Accepted 11 April 2018; Published 17 May 2018

Academic Editor: Pavlos I. Lazaridis

Copyright (c) 2018 Shang Gao et al. This is an open access article distributed under the Creative Commons Attribution License, which permits unrestricted use, distribution, and reproduction in any medium, provided the original work is properly cited.

\begin{abstract}
In traditional airplane monitoring system (AMS), data sensed from strain, vibration, ultrasound of structures or temperature, and humidity in cabin environment are transmitted to central data repository via wires. However, drawbacks still exist in wired AMS such as expensive installation and maintenance, and complicated wired connections. In recent years, accumulating interest has been drawn to performing AMS via airborne wireless sensor network (AWSN) system with the advantages of flexibility, low cost, and easy deployment. In this review, we present an overview of AMS and AWSN and demonstrate the requirements of AWSN for AMS particularly. Furthermore, existing wireless hardware prototypes and network communication schemes of AWSN are investigated according to these requirements. This paper will improve the understanding of how the AWSN design under AMS acquires sensor data accurately and carries out network communication efficiently, providing insights into prognostics and health management (PHM) for AMS in future.
\end{abstract}

\section{Introduction}

In a typical commercial/military aircraft, the AMS includes safety-critical system (e.g., engine control system, flight control system) and nonsafety critical systems (e.g., structural and engine health monitoring system, cabin environmental control system, and inflight entertainment system) [1]. Traditionally, a large number of real-time sensors based on wired connections have served for current AMS. For instance, in the airbus A380, over 300 miles of cables consist of around 40,000 sensor connectors and 98,000 wires [2]. Wired system in AMS has distinct features as follows: (a) cable routing is quite a strict and complicated task. For example, the power routing and electrical signal routing should be physically separated to prevent routings from electromagnetic interference, impeding airline customization in the course of manufacturing. (b) The utilization of wire harness is limited in accessible sensor locations and harsh environmental condition. (c) Installation of longer wires in large-size structure is time-consuming and labor-intensive. (d) Degradation of wiring might contribute to flight mission unfinished or termination, even severe catastrophic failures.
According to a US Navy report, about 78 aircraft are made flight mission incapable due to wiring faults, resulting in over 1000 mission aborts each year [3]. Traditional wired AMS [4] suffers from many shortcomings mainly due to long wires which connect each sensor to a central unit.

Till now, the progress achieved in embedded sensors technologies and wireless data transmission has extended the monitoring capability of aeronautical structures, spacecraft and ground testing equipment, and cabin environment [5]. One major potential advantage of using AWSN is the reduction of weight and installation time of aircraft system, fuel consumption, maintenance, and overhaul. The AWSN provides a new approach to resolve many issues intrinsic to wire-based instrumentation, such as fuel efficiency, carbon emission, and flight mass [6, 7]. It has been proved that the AWSN can result in 90 lbs. weight reduction of Cessna 310R control systems, $267 \mathrm{lbs}$. weight saving for an SH60 military helicopter control system [8] and 240 pounds reduction of wires for Blackhawk helicopters [9]. Generally, the cabling planning tasks for one aircraft have a cost of 2,200 dollars per $\mathrm{kg}$ [10]. The adopted AWSN can reach the savings of 14-60 millions of dollars per aircraft [11]. 
An attractive use of AWSN in AMS is sensing. In aircraft, due to high angles of attack in takeoff/landing, sudden pilot manoeuvres, turbulence, wind gusts, and normal shock waves on the wing at transonic speed, boundary layer separation on the wings the occurs, resulting in the phenomenon of parasitic drag and stall. For this reason, deployment of wireless airflow control actuators on strategic locations especially wing for providing real-time airflow information and decision metrics to the local system sheds light on the construction of efficient closed-loop airflow control operation [12]. The AWSN can implement self-configuration, RF tolerance, and maintenance troubleshooting [13]. In critical applications, although wireless links cannot completely replace cables in light of the high reliability requirement, they can also function as redundant links, enhancing the reliability and flexibility of AMS. Also, the AWSN will increase the safety and system flexibility due to less complicated fault allocations processing and fire hazards than wiring system.

This idea of operating AWSN for AMS was initially introduced by researchers in the early 21 century and several researchers remarked the potential benefits of this technology over traditional AMS systems. Nevertheless, issues should be still underscored when these systems kept in a long-term operation. As a matter of fact, the first AWSN prototypes employed low-processing MCU coupled with low-resolution analog-to-digital converter (ADC) and low sampling rate, but the technique makes sophisticated sensing and conditioning elements available. Recently, a number of scholars have been dedicated to investigating key issues (e.g., powerful hardware prototype, network protocol, time synchronization, and passive sensing) in this disciplinary field, indicating that AWSN becomes increasingly practical in AMS [14-19].

Several well-known research institutes have invested adequate funds for AWSN based AMS. For example, the Wireless Interconnectivity and Control of Active Systems (WICAS) project funded by the Engineering and Physical Sciences Research Council (EPSRC) applies AWSN to aircraft wing active flow control [20]. The Flite Instrumentation Test Wireless Sensor (FLITE-WISE) project backed by the European Commission has developed AWSN to facilitate the continuous monitoring of European aircraft. The aim of the project is to move away from the unnecessary burden presented by wires, making aircraft maintenance more efficient [21]. The SAHARA project supported by ASTECH company is a French $\mathrm{R} \& \mathrm{D}$ project which targets wireless sensors applied on aircraft, helicopters, and space vehicles [22]. The United States Air Force has started the Advanced Subminiature Telemetry (ASMT) program in Florida at Eglin Air Force Base aimed at developing a AWSN system for aircraft ground and flight test monitoring [23, 24]. The Flyby-Wireless (FBW) Alliance led by NASA Langley Research Center announced to largely fund four research projects to apply AWSN for AMS [25, 26].

Rapid advances on composite materials and piezoelectric sensors have presented new opportunities to AMS, essential to make more comprehensive analysis for damage, impact, and crack monitoring [27]. Typically, the integration of piezoelectric sensors and AWSN has opened a new door for active AMS [28-30]. The simplicity, robustness, and potentially low cost of piezoelectric sensors determine the suitability of their embedment into aircraft composite structures, contributing to excite and sense Lamb waves as an online aircraft health monitoring method [31,32]. Depending on the capability of local signal conditioning, data processing and wireless communication and wireless piezoelectric node can locally carry out the interpretation and processing of Lamb wave, promising a real-time large-scale AMS [33].

This paper is organized as follows: Section 2 describes a brief context of AMS, AWSN, and the characteristics of AWSN from different aspects (accuracy, real-time, reliability, time synchronization, throughput, longevity, safety, and security). Section 3 presents the current platforms and network communication schemes of AWSN. Finally, potential paths for future development and main challenges are briefly outlined in Section 4 followed by the conclusions in Section 5.

\section{Context of AWSN}

In this section, the context of AWSN is discussed, from both a general and a specific perspective. Firstly, a brief description of AMS is presented to illustrate the role of AWSN in this novel concept and framework. Then, an AWSN diagram is introduced to show the structure of general AWSN in which the architecture of airborne wireless sensor nodes, communication networking, and node deployment are demonstrated. Finally, we summarize the characteristics required for AWSN to accommodate new requirements of AMS.

2.1. The Overview of $A M$. In this review, the AMS mainly includes airplane structural health monitoring (SHM) and airplane cabin environmental monitoring. The AMS collects data from various sensors deployed on airplane structures and installed inside airplane cabin, implementing structural health monitoring and cabin environmental monitoring, respectively. With the rapid development of new materials and advanced technology in airplane, the modem structures are becoming complicated increasingly. The airplane SHM, including Lamb wave damage detection technology, optical fiber based global condition perception, multisensor fusion detection technology, and structural health assessment, provides approaches for assessing the health condition and ensures the safety of the complicated structures. Especially, structural response is obtained through strain, vibration, ultrasound, and piezoelectric sensor online structural health monitoring on airplane structures. The response is used to evaluate structural health status and assess the residual life of the airplane structure, further to develop PHM. Overall, the SHM is aim to save money spent on maintenance or replacement and ensure the structure to operate efficiently during its whole intended life.

The airplane cabin full of a mixture of outside and recirculated air is a semienclosed structure. Generally, the airplane cabin is in a low humidity, low pressure dynamic condition. Additionally, various concentrations of ozone $\left(\mathrm{O}_{3}\right)$, carbon monoxide $(\mathrm{CO})$, carbon dioxide $\left(\mathrm{CO}_{2}\right)$, and other chemicals are generated and spread over the airplane cabin. Different locations (e.g., on the ground, in ascent, at cruise, or in 


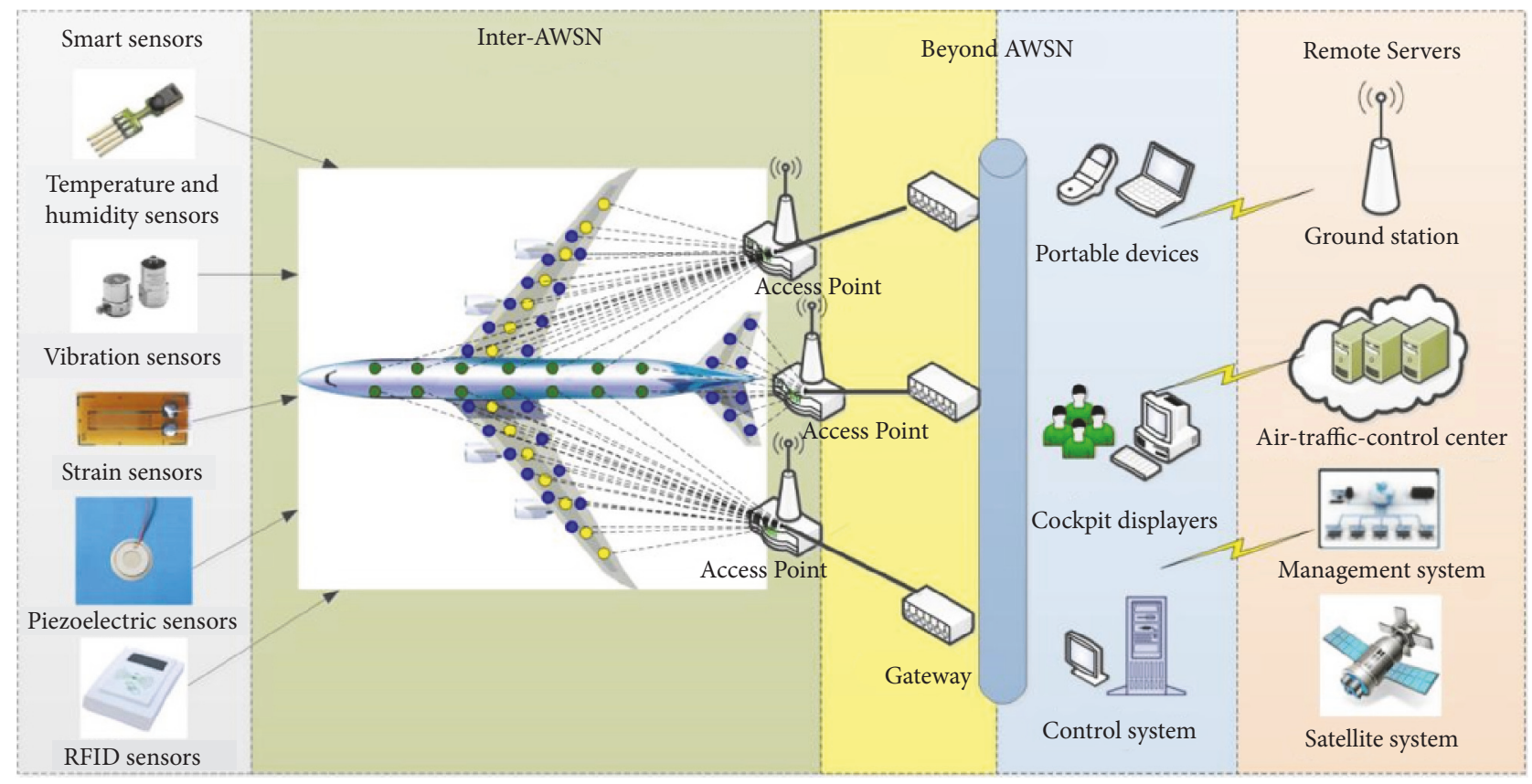

FIgURE 1: The airborne wireless network schematic diagram.

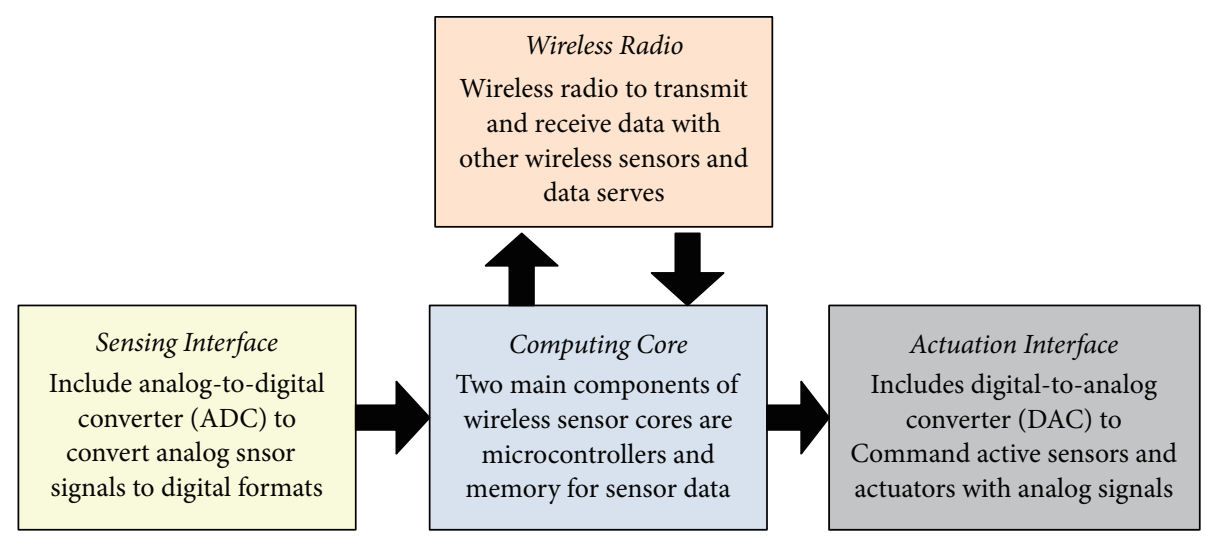

FIGURE 2: The schematic diagram of airborne wireless sensor board.

decent) of the airplane determine the level of contaminants invaded from outside sources. If the level of contaminants is not real-time monitored and adjusted timely, it is harmful and dangerous for passengers and crews.

2.2. The Overview of AWSN. To further illustrate the framework of AWSN, we will concentrate on wireless communication from the perspective of AWSN deployed in AMS. As shown in Figure 1, The AWSN communication system includes four components: smart sensors, inter-AWSN, beyond AWSN, and remote servers. Within AWSN, various smart sensors deployed on airplane connect to airborne wireless sensor nodes. It is clear that the AWSN is formed among all sensor nodes depending on wireless transceivers. Beyond AWSN, access point nodes and the gateway create a bridge to other networks in airplane such as portable devices, cockpit displayers, and control system. Finally, higher-level data applications, including satellite network, ground station, air-traffic-control center, and management system, are based on these specific networks. For communication and networks within an airplane, the AWSN has become a good complimentary network to an airborne wired communication network.

2.2.1. Airborne Wireless Sensor Nodes. The basic block of any wireless sensor network is the airborne wireless sensor board. The appropriate selection of board is favorable for the performance of wireless monitoring. As shown in Figure 2, typical airborne wireless sensor board consists of three or four functional subsystems: sensing section, computational core, wireless transceiver, and, for some, an additional actuation interface. 
TABLE 1: Comparisons of different Wireless Avionics Intra-Communications.

\begin{tabular}{|c|c|c|c|c|c|}
\hline Standards & Standard & $\begin{array}{l}\text { Max. } \\
\text { throughput }\end{array}$ & Frequency & $\begin{array}{c}\text { Free-space } \\
\text { range }\end{array}$ & Spectrum \\
\hline $\mathrm{WiFi}$ & IEEE 802.11 & $54 \mathrm{Mbps}$ & $2.4 \mathrm{GHz}$ & $150 \mathrm{~m}$ & Unlicensed \\
\hline Zigbee & IEEE 802.15.4 & $250 \mathrm{Kbps}$ & $868 / 915 \mathrm{MHz} / 2.4 \mathrm{GHz}$ & $300 \mathrm{~m}$ & Licensed \\
\hline Bluetooth & IEEE 802.15.1 & $24 \mathrm{Mbps}$ & $2.4 / 5 \mathrm{GHz}$ & $150 \mathrm{~m}$ & Unlicensed \\
\hline RFID & ISO/IEC24791 & $640 \mathrm{Kbps}$ & $125 \mathrm{kHz} / 13.56 \mathrm{MHz}$ & $10 \mathrm{~cm}-10 \mathrm{~m}$ & Unlicensed \\
\hline LoRaWAN & IEEE 802.15 .4 & $50 \mathrm{Kbps}$ & $433 / 780 / 868 / 915 \mathrm{MHz}$ & $14 \mathrm{Km}$ & Unlicensed \\
\hline SigFox & IEEE 802.15.4 & $100 \mathrm{Kbps}$ & $868 / 902 \mathrm{MHz}$ & $17 \mathrm{Km}$ & Unlicensed \\
\hline NB-IOT & IEEE 802.15 .4 & $200 \mathrm{Kbps}$ & $900 \mathrm{MHz}$ & $22 \mathrm{Km}$ & Licensed \\
\hline WirelessHART & IEEE 802.15 .4 & $250 \mathrm{Kbps}$ & $2.4 \mathrm{GHz}$ & $150 \mathrm{~m}$ & Unlicensed \\
\hline ISA100.11a & IEEE 802.15 .4 & $250 \mathrm{Kbps}$ & $2.4 \mathrm{GHz}$ & $150 \mathrm{~m}$ & Unlicensed \\
\hline WiMAX & IEEE 802.16 & $300 \mathrm{Mbps}$ & $11 \mathrm{GHz}$ & $100 \mathrm{~m}$ & Unlicensed \\
\hline $60 \mathrm{GHz}$ & $60 \mathrm{GHz}$ & $3000 \mathrm{Mbps}$ & $5-7 \mathrm{GHz}$ & $10 \mathrm{~m}$ & Unlicensed \\
\hline UWB & UWB & $200 \mathrm{Mbps}$ & $3.1-10.6 \mathrm{GHz}$ & $10 \mathrm{~m}$ & Unlicensed \\
\hline WAIC & $\begin{array}{c}\text { C-Band } \\
\text { IEEE 802.15.4 }\end{array}$ & $250 \mathrm{Kbps}-200 \mathrm{Mbps}$ & $\begin{array}{c}\text { 2.2-3.4 GHz, } \\
\text { 4.2-4.5 GHz, } \\
\text { 5.0-5.15 GHz, } \\
\text { 5.091-5.15 GHz }\end{array}$ & $100 \mathrm{~m}$ & Unlicensed \\
\hline
\end{tabular}

The computation core is the primary difference between a airborne wireless sensor board and its wire-based counterpart. The presence of a microcontroller unit (MCU) allows for onboard data processing, data storing, and preparing for communication. To fulfill these tasks, the measured data and executable program (such as damage detection routines) are embedded in random access memory (RAM) and read only memory (ROM), respectively. The size (in bits) of internal data bus for microcontroller is classified as 8-, 16-, or 32bits, determining processing speed and power consumption. Many different memory sizes and employed algorithms are commercially available, which are tailored in conformity with the particular monitoring activity to be performed.

The sensing section is dedicated for converting ana$\log$ output into a digital representation that can be handled by digital electronics. Some typically used sensors for AMS application include strain gauges, temperature sensors, accelerometer and piezoelectric sensors. Many sensing sections integrate more than one type of sensing elements, while others incorporate one sensor concentrating on one kind of physical quantity for accuracy and power-saving reasons. Usually, the section includes amplifier, linear, compensator, and filter. The sensing resolution relies on the ADC effective number of bits and measurement range in Volts, coupled with sensitivity of sensors. For most AMS applications, ADC resolution of 16 bits or higher is preferred for detecting signals. For example, generally, low sampling rates (e.g., less than $500 \mathrm{HZ}$ ) are adequate for aircraft structural health monitoring. However, wireless sensors are increasingly investigated for applying in acoustic and ultrasonic NDE; therefore, there has been a growing desire for higher sampling rates in excess of $500 \mathrm{~Hz}$.

The presence of radio frequency ( $\mathrm{RF}$ ) communication allows each board to interact with other nodes and to forward sensing data. For this reason, more stress on effective communication needs to be laid for the sake of AMS's reliability and high-performance transmission. This is particularly true as high data sampling rate, high fidelity sensing, high transmission rate, and large transmission range are often involved in AMS. RF communication is real challenge on aircraft structures made of composite or steel components.

The last subsystem would be the actuation interface in which the core element is the digital-to-analog converter (DAC). It allows converting digital data generated by MCU into a continuous analog voltage output for exciting active sensors (e.g., piezoelectric elements) interplayed with the physical structures. Actuators and active sensors installed on physical system can both be handled by an actuation interface.

2.2.2. Communication Networking. In Table 1, thirteen WSNs standards including WiFi, Zigbee, Bluetooth, RFID, LoRaWAN, SigFox, NB-IOT, WirelessHART, ISA100.1la, WiMax, $60 \mathrm{GHz}$, UWB, and Wireless Avionics IntraCommunications (WAIC) have been listed. The comparisons of parameters such as the standards, working frequency, maximum range, and maximum throughput are also listed. It is evident that most standards share the same framework (IEEE 802.11.15.4) and radio frequency. Nowadays, as a promising standard based on IEEE 802.11 , WiFi has become another option in WSN. However, WiFi is not designed for AMS applications. Recently, the civil aviation industry is developing new standards such as WAIC for airplane. The WAIC is to provide highly reliable short-range radio communications between avionic systems and onboard subsystems in the same aircraft. In 2015, the World Radio Conference [47] proposed a new spectrum band of $5 \mathrm{GHz}$ [48] or 4.2 to $4.4 \mathrm{GHz}$ [49] for the WAIC. The WAIC systems can also use parts of IEEE 802.15.4 standard [50]. Compared to $5 \mathrm{GHz}$, the frequency band of $4.2 \mathrm{GHz}$ to 
4.4 GHz is more suitable for the WAIC as the WAIC in $5 \mathrm{GHz}$ and radio altimeter share the same frequency [51]. Till date, the frequency of 4.2 to $4.4 \mathrm{GHz}$ has emerged as a promising band for WAIC communications. In addition, the electromagnetic waves from wireless airborne devices inside internal airplane suffer from high attenuation as they penetrate complex metallic and composite spaces. In Table 1, the values of free space range in different wireless standards do not take propagation path loss into account. The AWSN has inherited many features from WSNs, especially communication protocol. Thus, all protocols can be applied in AWSN theoretically. However, due to the particular features of airborne environment, some new constraints and requirements in Section 2.3 will be demonstrated for AWSN.

2.2.3. The Deployment of AWSN in AMS. General aircraft body is consisted of left and right wing, cockpit or cabin, engine, vertical tail, left and right horizontal stabilizer, landing gear, front, middle, and rear sections which are installed in subsystems of aircraft. Due to the dispersing deployment characteristic of the subsystems, cluster-star network topology is more suitable for AWSN in AMS [78]. Figure 1 illustrates how the WSN is deployed inside cabin, fuel tank, on the wings and other sections of the airplane with clusterstar topology. To meet the requirements of AMS, one or more clusters are constructed in each subsystem or respective region of aircraft body, and cluster head and sensor nodes in each cluster formed cluster topology. The deployment of sensor nodes at certain optimum locations inside airplane enumerates as follows:

(1) Fuel Tank. The sensor nodes are deployed inside fuel tanks which are located in the wings and tail of the airplane to measure the level of fuel.

(2) Exhaust. Sensors placed inside the exhaust would monitor whether any obstructions exist in it.

(3) Wheels. The routine examination for health and condition of the wheels should be implemented before takeoff and after landing of aircraft. Moreover, the wheels might be also damaged while the aircraft is on the runway or in the air.

(4) Engine. The engine is the "heart" of an airplane, which should be monitored in real-time. Overheating or physical damage of the engine is harmful for airplanes. Most severe catastrophic failures even airplane crashes are associated with the safety of engine. Thus, sensor nodes installed in and around the engine would monitor temperature and state of the engine surrounds and all components.

(5) Wings. Wings in the aircraft are always exposed to corrosion, impact, and crack damage due to external various complicated climatic environments. Sensor nodes installed in the wings would monitor vibration or strain arising from them to diagnose or forecast the localization, severity of them.

(6) Fire and Safety. Certain areas inside the airplane such as the aircraft cabin and the passenger area carry items

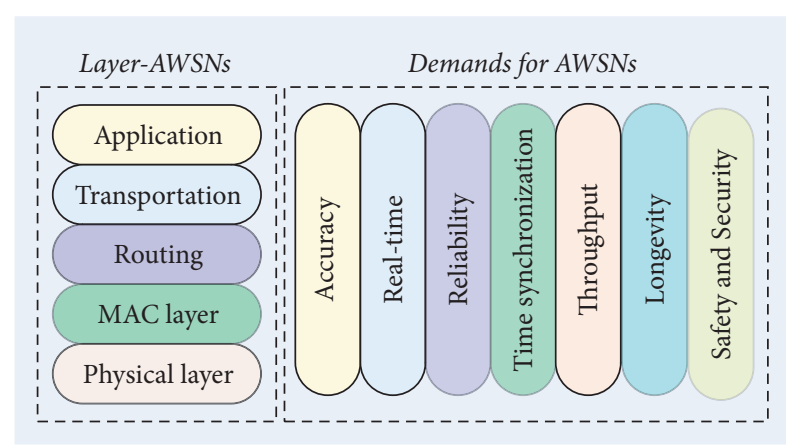

FIgURE 3: The characteristics for AWSNs.

like luggage, cockpit, the kitchen, passenger section, and the cargo, where smoke sensor nodes might detect fire indication and send alerts through the AMS.

2.3. The Requirements of AWSN for AMS. The AWSN has some requirements particularly for the environment of AMS. Generally, the architecture of ordinary WSN is constituted with different layers including application, transporting, routing, medium access control (MAC), and physical layer. However, this traditional layer architecture cannot satisfy the requirements for AWSN applied in AMS. The requirements cover accuracy, real-time, reliability, time synchronization, throughput, longevity and safety and security in terms of their constraints, challenges, and design goals for realizing AWSN, as shown in Figure 3. To meet these requirements, the design of platforms and MAC scheme for AWSN needs to be considered throughout all layers in Section 3.

2.3.1. Accuracy. The accuracy of acquired data is an important data quality aspect. In the AWSN, data accuracy is directly related to the accuracy of the airplane prognostics and health management, such as fault detection, fault isolation, fault prognosis, and prognosis of the remaining life. Additionally, the accuracy of acquired data affects the safety of airplane, the economic profits, and flight efficiency. In AWSN, the context is also associated with time synchronization, node number, hop number, and sampling rate.

2.3.2. Real-Time. WSNs have been applied in smart plants, industrial environment monitoring, and automation factories for low latency wireless communication, which sets an example for the AWSN. Real-time performance is an important issue for the AWSN. Communications between wireless nodes require latency to improve productivity. However, different applications may have different real-time requirements. Table 2 shows the latency requirements for different AMS subsystems. Flight motion control systems have the fastest real-time requirement $(<60 \mathrm{~ms})$, while monitoring systems tolerate the largest latency value ( $<1 \mathrm{~s})$. Many researchers have presented methods to achieve good realtime performance from different views and layers of wireless networks. Most of these studies have achieved low latency by improving the routing, MAC, or transport layers. Section 3.2 
TABLE 2: Real-time requirements in different AMS.

\begin{tabular}{lc}
\hline AMS & Latency \\
\hline Automation system & $<3 \mathrm{~ms}$ \\
Environmental monitoring system & $<1 \mathrm{~s}$ \\
Flight motion control system & $<60 \mathrm{~ms}$ \\
Wing control & $<100 \mathrm{~ms}$ \\
\hline
\end{tabular}

gives a summary of different communication schemes for real-time requirements.

\subsubsection{Reliability}

Path Loss. The wireless airborne devices employed inside airplane forward structural or environmental data to data aggregation center. Unfortunately, the electromagnetic waves from devices inside internal airplane should penetrate complex metallic and composite spaces. For instance, wireless propagation in airplane wing suffers from high attenuation as it goes through wing relief holes and spaces between wing structures including skin, spar, stringers, and ribs. However, path loss models for airplane wing environment link have been seldom investigated. It is challenging to get complete wireless link budget due to the high metallic feature of airplane wing. Due to the particularity of airplane wing environment, traditional path loss models for building or outdoor environments (e.g., alfish-Ikegami [79], KeenanMotley [80], and Turkmani [81]) are unsuitable for empirical path loss of airplane wing.

Signal Interference. The wireless airborne devices inevitably face interference due to elements of the harsh environment such as heavy dust, vibration, heat, freeze, uncertain temperature and humidity, bad weather in upper air, and other RF signals [82], which contravene wireless communication principles. Because personal wireless mobile devices such as mobile phones and laptops are not allowed to operate in airplane cabin, they will not produce signal interference. In addition, as Zigbee, Wi-Fi, UWB, and Bluetooth protocols can be adopted in AMS, they also have to coexist with each other. If the AWSN has only one working channel, it usually suffers from frequency interference and packet errors. In external aircraft, the signal interference sources are generally from air-ground $(A / G)$ communication system, airair (A/A) communication system, satellite-based communication systems, aeronautical Mobile Airport Communication System (AeroMACS), and other communication systems [83]. The A/G communication systems use HF, VHF, and $\mathrm{L}$ bands for data transmission. The A/A communication systems adopt $\mathrm{HF}$ band. Also, the satellite-based aeronautical communication systems usually use VHF, $\mathrm{X}, \mathrm{Ku}$, and $\mathrm{Ka}$ bands. As shown in Table 3, most frequencies of S-Band and C-Band in AWSN and WAIC coexist with most bands in A/G communication system, A/A communication system, satellite-based communication systems and AeroMACS [84]. However, the overlapping bands between AWSN, WAIC, and other systems should also be underlined. For instance, the onboard GNSS inside aircraft conveys the aircraft position and speed to Extended Squitter (ES) signals and sends them by $A / G$ communication system. The ground stations receive the ES signals, report to air traffic controller, and then respond to aircraft $\mathrm{A} / \mathrm{G}$ communication system. The ES operating in the $1090 \mathrm{MHz}$ band is vulnerable to cochannel interference from the AWSN. Moreover, the WAIC and aeronautical radio altimeters (RAs) share the band of 4.2 to $4.5 \mathrm{GHz}$, as well as the WAIC, and airport surface applications share the band of $5.091-5.15 \mathrm{GHz}$, which has the risk of harmful mutual interference [49]. The RAs are utilized to provide accurate and reliable measurements of the minimum distance to the earth surface. They operate in the process of flight or in the situation where the aircraft is located on ground or taking off. Table 3 summarizes all aeronautical spectrum bands and their services.

Packet Loss and Bit Error. The reliability of the AWSN for AMS is an important evaluation index. However, the harsh aircraft environment of AMS can introduce more interference and increase the packet loss rate (PLR) and the bit error rate (BER) of the AWSN. Retransmission mechanism is an approach generally to handle with these problems, which also increases the latency. This is an issue for AWSN and therefore some researchers have proposed efficient algorithms to increase network reliability in Section 3.2.

2.3.4. Time Synchronization. Each airborne wireless sensor node has its own local clock, which is not initially synchronized with other nodes. Two jitters, namely, temporal jitter and spatial jitter, occur inside node and between different nodes, respectively, due to variation in oscillator crystals. Time synchronization errors between different devices mean that obtaining the proper mode shapes without deviating from reality or theoretical calculations of structure is impossible [85]. How synchronization errors affect the process of obtaining mode shapes and why the synchronization error should be below $1 \mathrm{~ms}$ in order to get valid data are studied in [86]. Synchronization errors contribute to the inaccuracy of synchronized data acquisition and unstable longtime operation of the AWSN. Furthermore, the precision of time synchronization affects the validity of structural analysis and the accuracy of structural damage diagnosis [87]. In AWSN, the time synchronization error should be controlled within microsecond. At present, many researches on clock synchronization of WSNs, such as TPSN [88], RBS [89], and FTSP [90], have the synchronization accuracy of 20 us approximately. However, data sampling rate is not considered in these methods. If the data sampling rate (e.g., vibration) increases in AWSN, airborne wireless sensor nodes spend more time for data acquisition, contributing to the decrease of synchronization accuracy [91]. To tackle this limitation and ensure the synchronization accuracy, systems have to reduce the number of nodes at the expense of network throughput performance. Therefore, in airplane SHM, the goal is to develop a complete wireless measurement system with appropriate time synchronization method in which the accuracy is always less than 20 us', the number of nodes is not limited, and reliability is no longer an issue. 
TABLE 3: The overview of all aeronautical spectrum bands and their services.

\begin{tabular}{lcc}
\hline Band & Frequency & Service \\
\hline HF & $3-30 \mathrm{MHz}$ & $\begin{array}{c}\text { A/G communication system, A/A communication system } \\
\text { A/G communication system, satellite-based aeronautical } \\
\text { communication system }\end{array}$ \\
L-Band & $117.95-137 \mathrm{MHz}$ & $\begin{array}{c}\text { AWSN } \\
\text { A/G communication system, Extended Squitter (ES) signals, and }\end{array}$ \\
X-Band & $960-1215 \mathrm{MHz}$ & \\
Ku-Band & $1350-1400 \mathrm{MHz}$ & Satellite-based aeronautical communication system \\
Ka-Band & $10.4-14.3 \mathrm{GHz}$ & AWSN, WAIC \\
S-Band & $15.15-15.35 \mathrm{GHz}$ & WAIC, Radio Altimeters \\
C-Band & $26-40 \mathrm{GHz}$ & WAIC \\
C-Band & $2.2-3.4 \mathrm{GHz}$ & Airport surface applications, WAIC.
\end{tabular}

2.3.5. Throughput. For AWSN application, it is required to improve the network throughput for collecting large amount of acquired data in AMS. Theoretically, the baseline throughput between two nodes with single-radio at IEEE 802.15.4 band is $250 \mathrm{Kbps}$. Furthermore, Osterlind and Dunkels proved that the maximum data throughput at IEEE 802.15.4 band is $225 \mathrm{Kbps}$ [92]. If the packet copying is considered between the transceiver and the microcontroller during real data sampling and sending procedure, the maximum data throughput is $140 \mathrm{Kbps}$ approximately. Furthermore, if the network communication protocol is also considered when multiple nodes are accessed into network, further decrease of data throughput will result in the time delay of transmission, thus weakening the real-time performance of AWSN. For instance, $40 \mathrm{MB}$ data acquired in AWSN will take 18 hours approximately to be transferred to data aggregation center in light of communication protocol. The multichannel communication can improve the data throughput by transferring data in different communication channels [93, 94]. However, most multichannel communications in the single-radio sink node depend on one radio module. Some researchers investigated multichannel communication by employing multiradio sink node. In the multiradio sink node, switching among different channels is avoided and data flowing in all radios can be received parallel from senders, which can greatly improve data throughput.

2.3.6. Longevity. Many studies have pointed that WSNs are still constrained by energy limits because most of airborne wireless sensor nodes are usually battery-powered. In this case, it is impractical to replace and recharge a large number of batteries. Furthermore, in AWSN, batteries not only provide energy for wireless communication but also for mechanical systems, so the energy limitation is a critical challenge for prolonging network life. Typical WSN deployments employ battery-powered nodes. These onbattery nodes are usually deployed randomly and widely. It is impossible for them to be recharged once deployed. We should try our best to provide staple networking functionality within limited energy budgets and reduce their energy consumption by using innovative routing algorithm. In this section, we will concentrate on two aspects to prolong the operation of AWSN and discuss the longevity.

The first approach to ensure longevity of AWSN is efficient energy management and conservation. Energy efficient strategies have been implemented in different layers. For instance, in the physical layer, unnecessary actions can be reduced, and the physical parameters can be optimized to achieve strong power-saving performance. In the application layer, several useful methods such as eventdriven techniques, application-driven techniques, and efficient data/messaging can be used to decrease energy consumption. Additionally, as for networking and communication, the protocols of MAC routing can be designed to reduce the energy consumption. For instance, sleeping and working mechanism is adopted to achieve good energy conservation.

Energy harvesting and transferring power wirelessly are other approaches to improve the longevity of AWSN, which redefine the traditional design of battery-operated AWSN. The WSNs coupling with ambient energy harvesting can prolong the system's lifetime or possibly enable perpetual operation. Meanwhile, the self-powered nodes have longer life-time for routing and path selections for data transmission than on-battery nodes. Many researchers have studied energy harvesting by exploring environmental energies such as solar, vibration, wind, and microwave [54-59] to increase energy efficiency and save power. However, the self-powered nodes cannot be rearranged as their locations are presented by AWSN requirements. The transferring power wirelessly has the ability to move energy across space, allowing for exploiting abundant energy sources available at places other than the locations of sensing. Moreover, the transferring power wirelessly balances energy across the AWSN by distributing the available energy from energy-rich locations to energy-poor locations. Table 4 summarizes sources of energy harvesting for AMS in more detail.

2.3.7. Safety and Security. Safety and security are an important consideration in safety-critical avionics applications. In both wired network and AWSN, they should be considered 


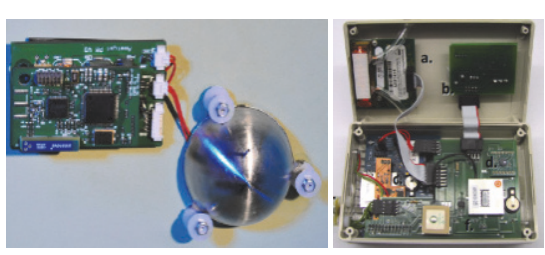

D.Samson

(a)

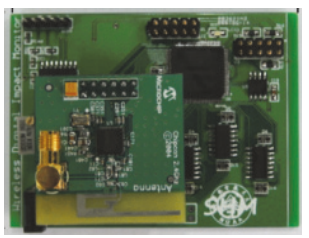

Liu

(e)

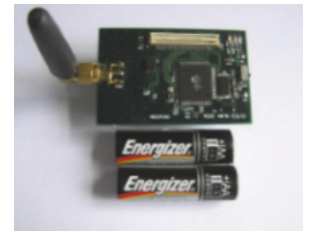

$\mathrm{Wu} \mathrm{J}$

(f)

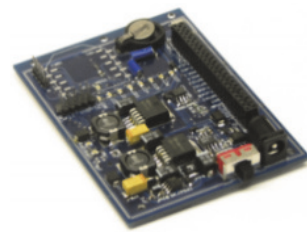

POOK M

(b)

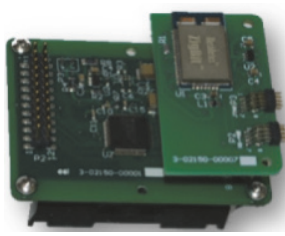

Demo J

(c)

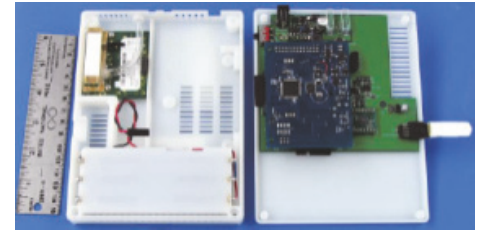

Kiepert J

(d)

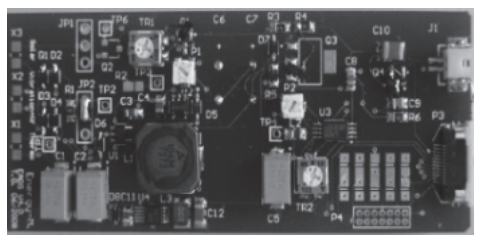

Becker

(g)

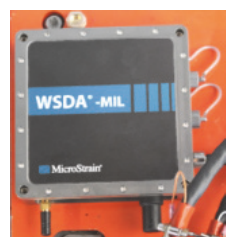

Arms

(h)

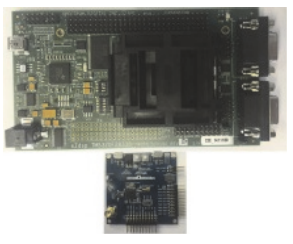

Shang Gao

(i)

Figure 4: Some of the proposed wireless sensor nodes: (a) Samson et al. [95]; (b) Pook et al. [35]; (c) Demo et al. [34]; (d) Kiepert et al. [36, 37]; (e) Liu et al. [38]; (f) Wu et al. [39, 40]; (g) Becker et al. [41]; (h) Arms et al. [42-44]; (i) Gao et al. [45, 46].

TABLE 4: Some sources of energy harvesting for AWSN.

\begin{tabular}{lc}
\hline Authors & Harvesting energy \\
\hline Samson et al. [52] & Thermoelectric \\
Lu et al. [53], Hart et al. [54], Hadas et al. [55], & Vibration \\
Arms et al. [43] & Solar \\
Siu et al. [56], Klesh and Kabamba [57] & Wind \\
Azevedo and Santos [58] & Microwave \\
Zhao et al. [59]
\end{tabular}

in the OSI protocol stack including physical, MAC, routing, transportation, and application layer. The AWSN is vulnerable to malicious attacks in all layers, and security vulnerabilities with these layers are separately protected at each layer.

Jamming [95] is the main attack in physical layer in WSN. A common mechanism against physical layer jamming attack in WSNs is spread spectrum communication such as FHSS, DSSS, and THSS. In MAC layer, the attack mainly includes MAC spoofing [96] and man-in-the-middle (MITM) [97] attack. Mac spoofing attack can alter the MAC address and code it into NIC card, making nodes take illicit activities. Another attack type, MITM attack, intercepts MAC address of the legitimate nodes by sniffing network traffic. It functions as relay between two victim nodes. In routing layer, IP protocol is in charge of data or packet delivery from the source to the destination through routers using IP addresses. The attacks in this layer include hijacking [98] and smurf attack [99]. IP hijacker controls the IP address of legal users, contributing to disconnection between legal users and network and finally establishing an illegal network. In smurf attack, a large number of ICMP packets are sent to the victim nodes which respond to ICMP requests. This attack overwhelms the victim network in this way. In transport layer, the TCP flooding attack [100] and UDP flooding attack [101] are implemented by sending large number of ICMP ping requests and UDP packets to victim nodes, respectively. The two flooding attacks contribute to the delay connection of victim to the target network. In application layer, several protocols such as HTTP, FTP, and SMTP are vulnerable to security attacks. Malware attack [102] includes the ways of Trojan horse, worms, key-loggers, and viruses. SQL injection [103] attacks network by acquiring unauthorized access to websites. Firewalls and antiviruses are required to counter these attacks. As shown in Table 5, we summarized various security attacks, their characteristic features, and countermeasures at different OSI layers.

\section{Current Platforms and Network Communication Schemes of AWSN}

In Section 2.3, seven requirements described above, accuracy, real-time, reliability, time synchronization, throughput, longevity, and safety and security, are closely related to each other. In AWSN, the main factors including hardware platforms and MAC schemes determine the performance of the seven indexes in AWSN. Therefore, in this section, we survey current hardware platform design for AWSN applications. Also, we provide a brief comparative study of emerging and existing network communication schemes development for AWSN in terms of seven requirements. The purpose of these surveys is to investigate how to design a feasible and highperformance AWSN for satisfying the requirements of AMS. Table 6 presents the key components like computing specifications, data acquisition, and wireless section of airborne wireless sensor node prototypes. Furthermore, in Table 7, analytical comparisons of characteristics features such as throughput, max sampling rate, time synchronization error, and target application are condensed. A few examples of the developed sensor boards are shown in Figure 4. 
TABLE 5: Characteristics and countermeasures of attacks in various OSI layers.

\begin{tabular}{lccc}
\hline Attacks & Characteristics & $\begin{array}{c}\text { OSI layer } \\
\text { affected }\end{array}$ & Countermeasures \\
\hline Malware attack & Trojan horse, worms, key-loggers, and viruses & Application & Firewalls and antiviruses \\
SQL injection & Acquiring unauthorized access to websites & Application & Firewalls and antiviruses \\
TCP flood & Sending massive ping requests & Transport & Reducing packets response \\
UDP flood & Sending massive UDP packets & Transport & Reducing packets response \\
IP hijacking & Legal users IP address impersonation & Routing & Firewalls \\
Smurf attack & Sending massive ICMP requests & Routing & Reducing packets response \\
Mac spoofing & MAC addresses falsification & MAC & ARP packets \\
MITM attack & Communicating nodes impersonation & MAC & Virtual private networks \\
Jamming attack & Interrupting legal data transmission & Physical & Spread spectrum techniques \\
Denial of service & Sending abundant packets & Physical & Temper-proof packaging \\
\hline
\end{tabular}

3.1. Current Existing Platforms. In 2007, Zhao et al. [66, 67] investigated a wireless piezoelectric sensor/actuator platform for real aluminum aircraft wing health monitoring. The platform's computational core consisted of a C8051F120 MCU and ADC chip ADC1173 having 8 bit resolution and sampling rate of 10 MSPS, while it used LINX ES Series transceiver for $\mathrm{RF}$ transmission. A customized conditioning board generated $70 \mathrm{~V}$ peak-to-peak tone-burst signals ranging from $300 \mathrm{KHz}$ to $400 \mathrm{KHz}$ which was fed to PZT transducers for producing ultrasonic guided waves. The total power consumption of board was close to $203 \mathrm{~mW}$ (i.e., $55 \mathrm{~mW}$ for the RF transmitter, $33 \mathrm{~mW}$ required by the $\mathrm{ADC}$ ).

From 2007 to 2009, Wu et al. [39, 40] worked on developing a low cost wireless sensor node for aircraft structural health monitoring. The system incorporated 8 bit ATMEGA128 MCU and TI CC1000 RF transmitter. The conditioning board equipped a $10 \mathrm{bit}, 8$-channel, and low pass-filter AD623 chip providing input offset drift as low as $2 \mu \mathrm{V} /{ }^{\circ} \mathrm{C}$ and maximum sampling rate of $15 \mathrm{KSPS}$. The sensing element selected was P3500 strain indicator having a resolution of $1.32 \mathrm{~V} / \mu \varepsilon$. In 2009 , to further validate the reliability of the star-topology WSN, recorded strain data from aircraft fore-undercarriage strength testing system were implemented in Aircraft Strength and Research Institute of China [63].

In 2008 and 2009, Arms et al. [42, 43] adopted commercially available Agile-Link ${ }^{\mathrm{TM}}$ family nodes from well-known Microstrain Inc. [104] including SG-Link ${ }^{5}$ (Wireless Strain Node) which is used for the strain measurement of a Bell M412 helicopter in USA Army. In 2011, Arms et al. [44] further explored time synchronized algorithm for helicopter flight test and addressed tradeoff between wireless link quality, and power consumption, varying sampling frequency from $32 \mathrm{~Hz}$ to $4096 \mathrm{~Hz}$, acquisition channels and network size.

Generally, the WSNs for AMS mostly relied on active RF transmission. Some researchers described specific wireless passive devices under harsh conditions in aerospace vehicles. In 2011, Elmazria and Aubert [105] proposed small, simple and robust, and passive surface acoustic wave (SAW) device which is capable of enduring extreme and harsh conditions such as high level of radiation and electromagnetic interference, with temperatures up to $1000^{\circ} \mathrm{C}$. In 2013, a series of NASA's robust passive chemical wireless sensors (e.g., MEMS, SAW, RFID) [106] presented for aerospace vehicles from Space Shuttle, HyperX, and Helios would withstand high temperature above $1000^{\circ} \mathrm{C}$.

In 2012, as for online aircraft impact damage monitoring, Delebarre et al. [64] sought to study a piezoelectric WSN with energy harvesting ability, integrating a low-power consumption 8051 microcontroller for detecting whether voltage value arising from impact reaches threshold and WIFI module for Lamb wave transmission. Liu et al. [38] and Yuan et al. [107] further explored a light-weight $(120 \mathrm{~g})$ and low-power $(100 \mathrm{~mW})$ piezoelectric-based wireless digital impact node (WDIM) for damage subregion location. In this study, the wing box specimen for WDIM performance testing had the dimension of $1000 \times 1800 \times 200 \mathrm{~mm}^{3}$, with $24 \mathrm{PZT}$ sensors connected to WDIM covering monitoring range of $450 \times$ $750 \mathrm{~mm}^{3}$.

In 2016, starting from previous work proposed by Yuan et al. [62], Gao et al. [45] firstly developed a new double-radio relay node (D-RRN), extending the monitoring distance without degradation of wireless link quality. The M-RSN [62] and D-RRN used in an multihop WSN allowed for achieving maximum data throughput rate of $861.6 \mathrm{Kbps}$ for aircraft health monitoring system. For proving the performance, reliability, and synchronization precision of the WSN, 28 sensor nodes deployed on aircraft wing box and an UAV composite wing was capable of implementing strain sensor data acquisition on 7 simultaneous communication channels. A comparison of the strain results from evaluation with counterparts obtained using wired optic strain in respective location showed a maximum difference of $1 \%$ in the two sets of data.

Due to minor damage detection ability of Lamb wave produced by piezoelectric sensors for AMS, piezoelectric sensors showed great potential promises for online aircraft structural health monitoring. For this reason, Gao et al. [46] proposed new wireless piezoelectric sensor node made of 


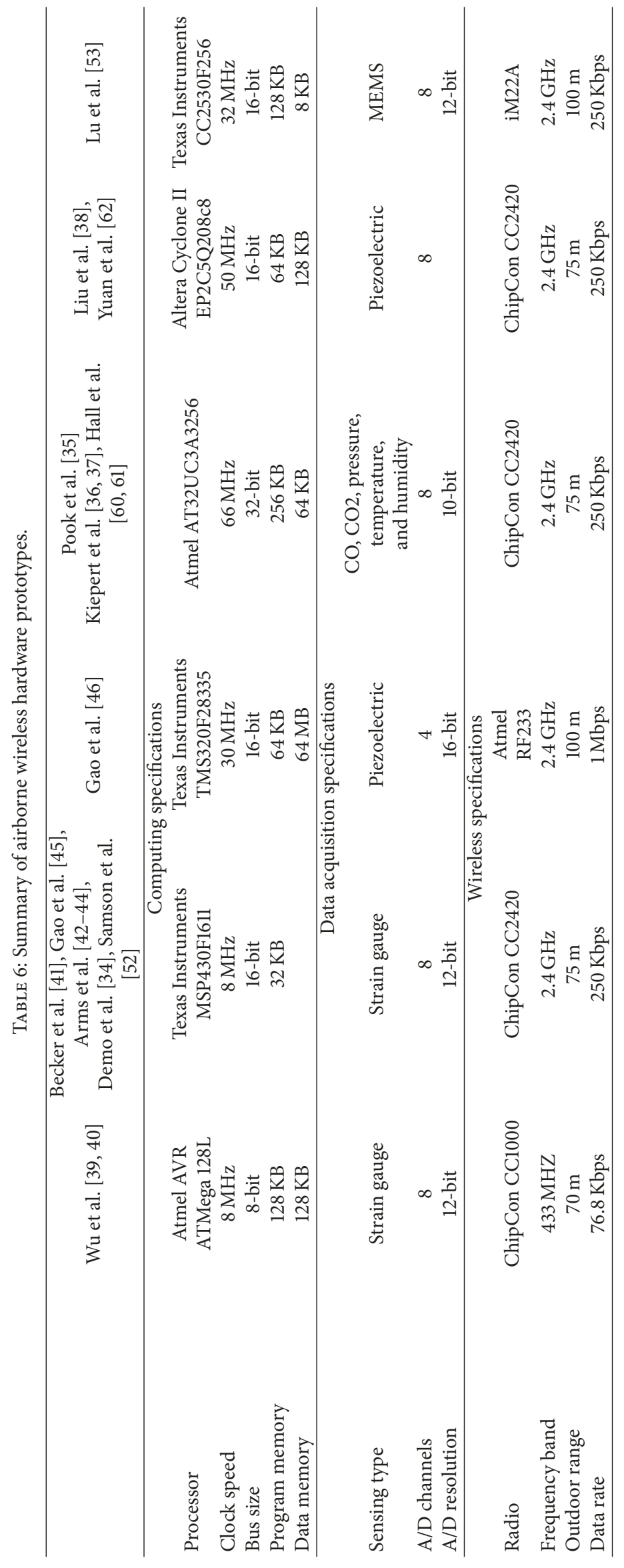


TABLE 7: Comparison of the characteristics features for airborne wireless prototypes.

\begin{tabular}{|c|c|c|c|c|}
\hline Study (-) & $\begin{array}{l}\text { Throughput } \\
\text { (Kbps) }\end{array}$ & $\begin{array}{l}\text { Max. sample } \\
\text { rate }(\mathrm{Hz})\end{array}$ & $\begin{array}{l}\text { Synchronization } \\
\text { accuracy (us) }\end{array}$ & Target application (-) \\
\hline Wu et al. [39] & 250 & 32 & - & Strain monitoring for aircraft carbon fiber reinforced plastic wing \\
\hline Wu et al. $[40,63]$ & 250 & 32 & - & box \\
\hline $\begin{array}{l}\text { Liu et al. [38], } \\
\text { Yuan et al. [62] }\end{array}$ & 250 & - & - & Impact monitoring for aircraft wing box \\
\hline $\begin{array}{l}\text { Delebarre et al. } \\
{[64]}\end{array}$ & - & - & - & Impact monitoring for aircraft wing box \\
\hline Gao et al. [45] & 909 & 32 & 50 & $\begin{array}{l}\text { Strain monitoring for aircraft wing box and an UAV composite } \\
\text { wing }\end{array}$ \\
\hline Gao et al. [46] & 2000 & 10000 & - & Aircraft aluminum plate \\
\hline Loo et al. [65] & 250 & - & - & Aircraft corrosion monitoring \\
\hline $\begin{array}{l}\text { Pook et al. [35] } \\
\text { Kiepert et al. } \\
{[36,37]}\end{array}$ & 250 & - & - & Aircrait corrosion monitormg \\
\hline Becker et al. [41] & - & 40000 & - & Laboratory environment \\
\hline Zhao et al. $[66,67]$ & 250 & 15000 & - & Ultrasound monitoring for aircraft wing inspection \\
\hline $\begin{array}{l}\text { Demo et al. [34] } \\
\text { Samson et al. [52] }\end{array}$ & 250 & 1000 & - & Aircraft corrosion monitoring \\
\hline Hall et al. $[60,61]$ & 250 & 50000 & - & Aircraft corrosion monitoring \\
\hline Arms et al. [42-44] & 250 & 512 & 39000 & Strain and vibration monitoring Bell Model 412 helicopter \\
\hline $\begin{array}{l}\text { Blanckenstein et al. } \\
\text { [68] }\end{array}$ & 250 & - & - & RSSI and BER for Airbus A330-300 \\
\hline Lu et al. [53] & - & 5300 & - & Lab environment \\
\hline
\end{tabular}

base board, radio board, and conditioning board. The base board installed a digital processor of 32-bit TMS320F28335 and 12-bit ADC with conversion rate of up to $10 \mathrm{MHz}$. The radio board integrated the commercially available Atmel SMART SAM R21 board with data rate of $2 \mathrm{Mbps}$. The conditioning board embedded an operational amplifier (Op AMPs) AD8608 with low noise ( $12 \mathrm{nV} /$ maximum), low bias current (1 pA/maximum), and low offset voltage $(65 \mathrm{uV} /$ maximum $)$ for the sake of maximizing the features of the 12-bit ADC. The tests were implemented on an aircraft aluminum plate of $2 \mathrm{~mm}$ thickness.

In 2016, Lu et al. [53] developed a WSN system powered by piezoelectric energy harvester, integrated CC2530F256 System-on-Chip (SOC) having enhanced 8051 core MCU architecture and low-power iM22A radio $\mathrm{RF}$ module. The SOC included 12-bit resolution multiplexed ADC with conversion rate of up to $4 \mathrm{KHz}$, while the sensor board installed three-axis LIS2DH accelerometer having a resolution equal to $9.65 \cdot 10^{-3} \mathrm{~m} \cdot \mathrm{s}^{-2}$, ultracompact digital pressure sensor (LPS25H), and digital humidity sensor (HDC1000). The accelerometer output signal fed 12-bit ADC having a resolution equal to $1.22 \cdot 10^{-3} \mathrm{~m} \cdot \mathrm{s}^{-2}$. The presence of sensors was used for correlating the effect of the aircraft environmental condition to the efficiency of energy harvester.

In 2009, Becker et al. [41] presented a kind of WSN node for aircraft strain measurement. The prototype, relied on TI MSP430 MCU having sampling rate of $40 \mathrm{~Hz}$ and ChipCon 2420 for RF transmission. The sensing element,
P3500 strain indicator, provided a resolution of $1.32 \mathrm{~V} / \mu \varepsilon$. A high-performance power management system was driven by thermoelectric energy harvesting device. All tests for the node were implemented in laboratory environment.

In 2009, Loo et al. [65] and Kiepert and Loo [36] developed 1st and 2nd generation sensor nodes for aircraft cabin environment monitoring in more than 200 commercial flights. They incorporated 8-bit Microchip PIC18F8722 with the million-instructions-per-second (MIPS) architecture and maximum operational frequency of $40 \mathrm{MHz}$. In 2011, starting from previous design, Kiepert et al. [37] and Pook et al. [35] updated the existing version with 32-bit AVR32 microcontroller having Dhrystone MIPS (DMIPS) architecture. Compared to the previous version, new prototype lifted computational capabilities and reduced power consumption. In laboratory test, two prototypes measure carbon dioxide (CO2), relative humidity, temperature, atmospheric pressure, and sound intensity. The system was tested over a period of 18 hours, close to the longest time during active commercial flight.

In 2010, Demo et al. [34] and Samson et al. [52] proposed Luna's wireless sensing platform for aircraft cabin environment monitoring. The based board embedded an ultralow-power MSP430 MCU with 12-bit A/D converter. The communication board installed Zigbee module based on IEEE 1451.5 protocol. Meanwhile the analog board provided transducer excitation and signal conditioning for all sensors, in stark contrast to previous versions used in aircraft cabin environment monitoring [34-37, 52, 65]. 
In 2012, Hall et al. [60, 61] developed an in-home air quality (IHAQ) node integrating different sensors in aircraft cabin environmental conditions related to air quality, health, and comfort and utilizing a Zigbee radio modules. For proving the capability of the IHAQ node, the prototype was tested during 7 hours in single-family home including three different sensor nodes deployed, showing that IHAQ node detected $\mathrm{CO} 2$, carbon monoxide (CO), humidity, temperature, pressure, and sound with resolution of $1 \mathrm{ppm}, 0.1 \mathrm{ppm}, 0.03 \%, 0.01^{\circ} \mathrm{C}$, $3 \mathrm{~Pa}$, and $0.02 \mathrm{dBA}$, respectively. These parameters met the requirements of the National Children's Study Program.

In 2014, Blanckenstein et al. [68] proposed a wireless sensor node consisting of 32-bit ARM CM3 EFM32G210F128 MCU and AT86RF233 2.4 GHz IEEE 802.15.4 transceiver having data rate of $2 \mathrm{Mbps}$ which is sufficient for transmitting high volume of sensor data. The SHT21 chip, humidity and temperature sensor, was chosen as the sensing element having resolution of about $0.04 \% \mathrm{RH}$ and $0.04^{\circ} \mathrm{C}$ for humidity and temperature, respectively. The prototype was evaluated with 500-node WSN deployment on Airbus A330-300 for measuring Received Signal Strength Indication (RSSI) and Packet Error Rate (PER).

\subsection{Comparative Studies of Existing Network Communica-} tion Schemes in AWSN. Efficient medium access control (MAC) scheme and routing protocol are highly required in AWSN. Many transmission protocols have been proposed for WSN. Demirkol et al. [108] listed the advantages and disadvantages of existing main MAC protocols (S-MAC, TMAC, DMAC, SCP-MAC, and LMAC), typically providing network communication and routing algorithm for WSN. Nevertheless, these protocols are proposed from a general insight into WSNs, rather than consideration for aeronautics specific application requirements. For instance, in S-MAC protocol, a packet delivery ratio (PDR) is evaluated less than 0.6 and time latency ranges from $3 \mathrm{~s}$ to $12 \mathrm{~s}$ under one packet for one node per second, which cannot satisfy the PDR and delay requirements of WSNs in AMS. Additionally, other requirements, high-precision time synchronization, and real-time data aggregation, demand further research on developing more suitable MAC protocol for AMS [33]. Typically, WSN MAC protocols served for AMS mainly fall into two categories: collision-free protocol (e.g., carrier sense multiple access (CSMA)) and contention-free protocol (e.g., time division multiple Access (TDMA) protocol).

The feasibility and reliability of collision-free protocol on the basis of IEEE 802.15.4 standard in wireless links for AWSN are fully considered [109, 110]. As for most collision-free protocols, Zigbee protocol is typically adopted as communication technique [73, 74]. Two standards [111], wireless HART from the HART Communication Foundation and ISA100.11a from the International Society of Automation (ISA), are introduced into WSN in aircraft application due to root from the IEEE 802.15.4 standard and its mature application in industrial control field. In 2011, by simulating Zigbee protocol with hierarchical WSNs topology on OPNET modeler platform, Notay and Safdar [75] found that data dropped, time delay, and throughput parameters are correlated with number of nodes and distance among nodes.
Results demonstrated that most MAC protocols based on CSMA still suffer from high collision rate and transmission unfairness between the nodes. To improve the performance of original CSMA protocol, researchers tried to employ intelligent algorithm for facilitating transmission efficiency operation of CSMA protocol. Barcelo et al. [72] developed channel and delay allocation (CDA) algorithm together with slotted CSMA protocol for aircraft conditioning monitoring, the verification of which was implemented by employing 80 nodes uniformly deployed along the body and the wings in 2 columns. Akkarajitsakul et al. [112] studied the feasibility of game theory coupled with CSMA protocol, for mitigating collision issues (e.g., packet loss, system throughput, as well as fairness). The GTMA protocol, a new algorithm proposed by Chowdhury et al. [71], also linked game-theoretic strategy to CSMA algorithm. Results from simulation indicated that GTMA protocol outweighed original CSMA protocol in terms of packet collisions, data throughput, and short-term fairness between the contending nodes. To fully investigate the implementation of game-based CSMA protocol in different real aircraft wing structures, Krichen et al. [69, 70] put forward various sensor deployment simulation models for specific types of aircraft wing and conducted respective network topology. Simulation framework constructed by Matlab software was performing in the deployment of 150 sensor nodes over aircraft wing of $40 \mathrm{~m} \times 10 \mathrm{~m}$ dimensions to monitor vibration and detect flutter phenomenon. They also remarked that game-based CSMA was superior to original CSMA protocol in respect to transmission delay, packet loss ratio, and data throughput.

Although a number of improved CSMA protocols have been proposed for WSN in AMS, they still suffer from many issues due to persistent collisions. The contention-free protocol such as TDMA algorithm provides better performance on latency, jitter, and spatial reuse than improved CSMA protocols. Meanwhile, to achieve high accuracy or high resolution of damage localization in aircraft structure, high-precision data synchronized acquisition in AMS should be underlined. To cope with these issues of star-cluster WSNs, Zhou and Jing [77] proposed MAC layer based on improved TDMA technique for AMS wherein the time slot length was flexibly allocated and adjusted according to sensor nodes traffic. Sensor nodes evaluated relative drift and offset and adjust their clock according to the clock embedded in synchronous message from cluster head at certain cycle. Simulations compared the performance of SMAC protocol, TDMA protocol, and modified TDM, proving that improved TDMA protocol has obvious advantages in terms of packet loss rate, time delay, and energy consumption. Furthermore, due to the linear multihop WSN topology commonly adopted in active airflow control, Omiyi et al. [113] developed TDMA-based hop-by-hop WSN convergecast scheduling strategies including serial line scheduling (SLS) and parallel line scheduling (PLS) algorithm, wherein sensor and actuator nodes were categorized into several linear clusters with each cluster consisting of $X$ nodes uniformly space along a straight line. The PLS was to maximize the number of linear clusters communicating in parallel, while SLS attempted to raise speed of per cluster sensor data 
delivery by increasing the number of nodes per cluster. To further boost the network performance of convergecast delay and tradeoff between latency and energy consumption, Dai et al. [114] proposed TDMA-based hybrid line, CSMA, with frequency division multiple access (FDMA) for large-scale aircraft health monitoring scheduling (HLS) protocol for air flow control. Results showed that HLS contributed to 15\% reduction in delay and energy saving at moderately high sensor densities. To access more nodes into network, in 2009 and 2011, Arms et al. [42-44] proposed a novel protocol integrating TDMA. 100 strain sensing nodes can access network at $100 \mathrm{~Hz}$ of sampling rate for each node on a singleradio channel. If FDMA was enabled and 16 channels in $2.4 \mathrm{GHz}$ were open, the system can support maximum of 1600 strain sensing nodes at $100 \mathrm{~Hz}$ sampling rate. Additionally, in 2014, Blanckenstein et al. [68] constructed a WSN with 500 sensor nodes deployed in Airbus A330-300. A robust and scalable TDMA protocol without multihop and ARQ-based retransmissions was proposed for guaranteeing maximum delay to maintain high channel utilization.

In Table 8, AWSN communication schemes are analytically compared based on types of platform, target application, reliability, energy efficiency, real-time transmission, topology, framework, and data flow as attributes.

\section{Research Challenges}

In recent years, more practical WSNs including system design and network protocol have been successfully proposed for AMS, evaluating safety of aircraft flight and engine control, the quality of aircraft cabin environmental condition, and the flexibility of aircraft structural health. Nevertheless, there is room for improvement of WSNs for AMS. More work is needed to allow WSNs to fulfill the requirements for largescale AMS. One important challenge researchers are now facing is turning the sensor node from ordinary data acquired device into online data processing intelligent "brain," making the WSN more powerful and efficient $[115,116]$. Additionally, emphasis should be laid on the improvement of power supply, data transmission reliability, and network bandwidth for WSN.

We should also identify how the achievement of highaccuracy time synchronization for large-scale AMS (especially in aircraft structural monitoring) is attained. The accuracy of time synchronization is closely correlated with network protocol and transmission bandwidth, sampling rate, and other factors. A large amount of data transmission results in network congestion, lower synchronization quality, and higher power consumption, whereas smaller data amount might reduce the accuracy of data analysis and increase network delay. It should be highlighted that tradeoff algorithm among the amount of data transmission, synchronization and network quality, and power consumption needs to be explored. More energy facilitates maximization of operating time for WSN. However, the battery life of WSN is too short for performing long-term AMS. Energy harvester [76] from ambient energy sources might be an effective approach and strategy to resolve this issue for powerconstraint sensor node. For abovementioned reasons, many researches will be committed to the construction of scalable networks by developing more effective energy harvests and control algorithms, reducing the amount of transmitted data and raising accuracy of network synchronization [117].

Nonsafety critical systems (e.g., wireless smoke and fire detection system, cabin emergency wireless-controlled lighting system) have been certified by the Federal Aviation Administration (FAA) which is typically operated in an unlicensed spectrum $[118,119]$. It has been simply proved that noninterference might exist between the regular wireless communication in aircraft electronic equipment and the wireless frequencies at $2.4 \mathrm{GHz}$ or sub-GHz band in AMS [120]. The recent study over the last few years focuses on the possible challenges and finds suitable solutions for Wireless Avionics Intra-Communications (WAIC) at $4.4 \mathrm{GHz}$ or $5 \mathrm{GHz}$. In future, more strict regulations are desired for executing to ensure that no interference existS among portable wireless electronic devices, equipped airplane radio transmitters, and WSN in AMS. Furthermore, future aviation standard must ensure that the WAIC, AWSN, and RAs operating cofrequency are able to coexist. With the rapid development of the Internet-of-things (IOT), it is predicted that 50 billion devices will be connected to the cloud (mainly via wireless links) by 2050 [121]. It is promising that new IOT systems such as IoT6 [122], the IoT ARM [123], and the ISO/IEC IOT architecture [124] with special frequency band will be possibly applied in the AWSN in future.

\section{Conclusions}

The AWSN is a promising technology which plays an increasingly key role in the AMS applications. However, few AWSN surveys consider its application background in AMS, so this is motivation of this review. The AWSN creates a new set of challenges in terms of accuracy, real-time, reliability, time synchronization, throughput, longevity, and safety and security. In this paper, a brief survey of AWSN and AMS including a range of areas from the general to the specific. The survey focuses on the prototype architecture and network communication schemes of the developed AWSN in AMS. It is outlined in this review that the node architecture and network communication schemes proposed in the early 21 st century are being developed and updated continuously, providing amounts of solutions for AMS. The first AWSN prototypes employed low-processing MCU coupled with low-resolution ADCs, but technology makes sophisticated sensing and conditioning elements available. Recent modifications and improvements made on board's MCU, RF transceiver, signal conditioning unit, and time synchronization show how WSN becomes mature gradually for applied in AMS. We further demonstrate that wireless piezoelectric platform based on Lamb wave algorithm shows great potential promises for online AMS. Besides, most traditional network communication schemes cannot satisfy all requirements of real-time AWSN in AMS. Intelligent algorithm (e.g., game theory) or machine learning might act as a new approach to resolve issues intrinsic to these protocols. These findings not only provide theoretical evidence and appropriate solutions to WSN design according to AMS requirements but also novel 


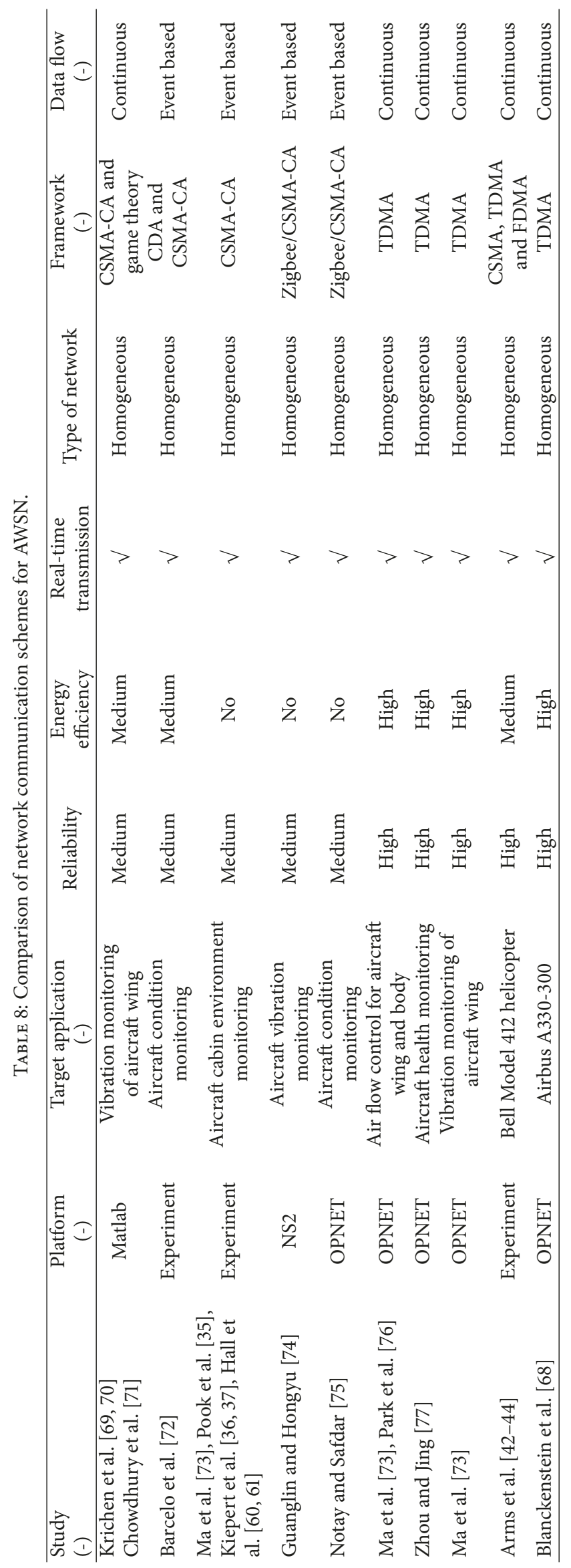


insights into airplane prognostics and health management (PHM).

\section{Conflicts of Interest}

The authors declare that there are no conflicts of interest regarding the publications of this paper.

\section{Acknowledgments}

This work was supported in part by Nanjing University of Science \& Technology under Research Start-Up grant (no. AE89991/032).

\section{References}

[1] S. Mazumdar and Q. Chen, "Response of contaminant detection sensors and sensor systems in a commercial aircraft cabin," in Proceedings of the 10th International IBSPA Conference, pp. 854861, Beijing, China, 2007.

[2] M. Heinen, “The A380 program," Global Investor Forum, 2006.

[3] J. Collins, "The challenges facing U.S. navy aircraft electrical wiring systems," in Proceedings of the 9th Annual Aging Aircraft Conference, 2006.

[4] D. Gorinevsky, G. A. Gordon, S. Beard, A. Kumar, and F. K. Chang, "Design of integrated SHM system for commercial aircraft applications," in Proceedings of the 5th International Workshop on Structural Health Monitoring, pp. 1-8, Stanford, Calif, USA, 2005.

[5] T. Vladimirova, C. P. Bridges, G. Prassinos et al., "Characterising wireless sensor motes for space applications," in Proceedings of the 2nd NASA/ESA Conference on Adaptive Hardware and Systems, pp. 43-50, Edinburgh, UK, August 2007.

[6] K. Sampigethaya, R. Poovendran, M. Li, L. Bushnell, and R. Robinson, "Security of wireless sensor network enabled health monitoring for future airplanes," in Proceedings of the 26th Congress of the International Council of the Aeronautical Sciences, pp. 2488-2497, Anchorage, Alska, USA, September 2008.

[7] K. Sampigethaya, R. Poovendran, L. Bushnell, M. Li, R. Robinson, and O. Lintelman, "Secure wireless collection and distribution of commercial airplane health data," IEEE Aerospace and Electronic Systems Magazine, vol. 24, no. 7, pp. 14-20, 2009.

[8] K. Kiefer, "Real-world experience in wireless instrumentation and control systems," in Proceedings of the CANEUS "Fly-byWireless" Workshop, 2007.

[9] D. Graham-Rowe, "Fly-by-wireless set for take off," New Scientist, vol. 203, no. 2724, pp. 20-21, 2009.

[10] M. Harrington, "Introduction to wireless systems in aerospace applications," in Proceedings of the CANEUS "Fly-by-Wireless" Workshop, Montreal, Canada, June 2009.

[11] O. Elgezabal Gomez, "Fly-by-wireless (FBWSS): benefits, risks and technical challenges," in Proceedings of the CANEUS "Flyby-Wireless" Workshop, pp. 14-15, August 2010.

[12] A. Arce, A. J. Del Real, C. Bordons, and D. R. Ramírez, "Realtime implementation of a constrained MPC for efficient airflow control in a PEM fuel cell," IEEE Transactions on Industrial Electronics, vol. 57, no. 6, pp. 1892-1905, 2010.

[13] B. Jing, J. Zhang, and Y. Sun, "Smart network sensor and wireless sensor networks," in Proceedings of the National Defense Industry Press, Beijing, China, 2011.
[14] S. Yuan, L. Qiu, and J. Wu, "Challenge in structural health monitoring of large aircraft development," Aeronautical Manufacturing Technology, vol. 22, pp. 62-67, 2009.

[15] T. R. Patterson, "Wireless sensor networks and data fusion for structural health monitoring of aircraft," Dissertations \& Theses-Gradworks, 2013.

[16] P. Wang, Y. Yan, G. Y. Tian, O. Bouzid, and Z. Ding, "Investigation of wireless sensor networks for structural health monitoring," Journal of Sensors, vol. 2012, Article ID 156329, 7 pages, 2012.

[17] R. K. Yedavalli and R. K. Belapurkar, "Application of wireless sensor networks to aircraft control and health management systems," Control Theory and Technology, vol. 9, no. 1, pp. 2833, 2011.

[18] H. F. Rashvand, A. Abedi, J. M. Alcaraz-Calero, P. D. Mitchell, and S. C. Mukhopadhyay, "Wireless sensor systems for space and extreme environments: a review," IEEE Sensors Journal, vol. 14, no. 11, pp. 3955-3970, 2014.

[19] R. D. Errico and L. Rudant, "UHF radio channel characterization for wireless sensor networks within an aircraft," in Proceedings of the 5th European Conference on Antennas and Propagation, pp. 115-119, Rome, Italy, April 2011.

[20] Roll-Royce Control \& Systems University Technology Centre, Wireless Interconnectivity and Control of Active Systems (WICAS), 2007, http://www.sheffield.ac.uk/systemsutc.

[21] http://cordis.europa.eu/news/rcn/122391_en.html.

[22] http://www.globalsys.fr/sahara/.

[23] S. Musteric and A. Berdugo, "The advanced subminiature telemetry system (ASMT): a wireless, network based, miniaturized instrumentation system," AIAA Journal, vol. 5, no. 32, pp. 123-135, 2013.

[24] F. Timothy, M. Devinder, and H. Iain, "F-35 Joint strike fighter structural prognostics and health management an overview," in Proceedings of the ICAF Conference, p. 1215, Springer, Dordrecht, the Netherlands, 2009.

[25] Aerospace safety enhancements at core of four projects selected by the CANEUS fly-by wireless consortium, http://www.caneus .org/fbw/content.aspx?id=2009_home.

[26] M. Pimprikar and I. Valenta, Fly-by-Wireless for Aerospace Vehicles, A Report of the CANEUS Fly-by-Wireless 2009 Workshop, CANEUS, Montreal, Canada, 2009.

[27] C. A. Paget, K. Tiplady, M. Kluge, T. Becker, and J. Schalk, "Feasibility study on wireless impact damage assessment system for thick aeronautical composites," in Proceedings of the 5th European Workshop on Structural Health Monitoring, pp. 16-85, Naples, Italy, 2010.

[28] H. Bai, M. Atiquzzaman, and D. Lilja, "Wireless sensor network for aircraft health monitoring," in Proceedings of the 1st International Conference on Broadband Networks (BroadNets '04), pp. 748-750, IEEE, San Jose, Calif, USA, October 2004.

[29] B. Nickerson and R. Lally, "Development of a smart wireless networkable sensor for aircraft engine health management," in Proceedings of the IEEE Aerospace Conference, pp. 3255-3262, Big Sky, Mont, USA, 2001.

[30] M. Reid, "Wireless eddy current probe for engine health monitoring," in Proceedings of the Quantitative Nondestructive Evaluation, pp. 414-420, Green Bay, Wis, USA, 2004.

[31] V. Giurgiutiu, J. M. Redmond, D. P. Roach, and K. A. Rackow, "Active sensors for health monitoring of aging aerospace structures," in SPIE's 7th International Symposium on Smart Structures and Materials and 5th International Symposium on 
Nondestructive Evaluation and Health Monitoring of Aging Infrastructure, vol. 3985 of Proceedings of SPIE, pp. 294-305, The International Society for Optical Engineering, Newport Beach, Calif, USA, June 2000.

[32] V. Giurgiutiu, A. Zagrai, and J. J. Bao, "Piezoelectric wafer embedded active sensors for aging aircraft structural health monitoring," Structural Health and Monitoring, vol. 1, no. 1, pp. 41-61, 2002.

[33] K. Bür, P. Omiyi, and Y. Yang, "Wireless sensor and actuator networks: Enabling the nervous system of the active aircraft," IEEE Communications Magazine, vol. 48, no. 7, pp. 118-125, 2010.

[34] J. Demo, A. Steiner, F. Friedersdorf, and M. Putic, "Development of a wireless miniaturized smart sensor network for aircraft corrosion monitoring," in Proceedings of the IEEE Aerospace Conference, pp. 1-9, Big Sky, Mont, USA, March 2010.

[35] M. Pook, S. M. Loo, and J. Kiepert, "Monitoring of the aircraft cabin environment via a wireless sensor network," in Proceedings of the 42nd International Conference on Environmental Systems, pp. 1-9, AIAA, San Diego, Calif, USA, July 2012.

[36] J. Kiepert and S. M. Loo, "A wireless sensor data fusion framework for contaminant detection," in Proceedings of the IEEE Conference on Technologies for Homeland Security (HST '09), pp. 214-220, Waltham, Mass, USA, May 2009.

[37] J. Kiepert, S. M. Loo, D. Klein, and M. Pook, "Wireless sensor networks for aircraft cabin environmental sensing," in Proceedings of the 41st International Conference on Environmental Systems, Portland, Oreg, USA, July 2011.

[38] P. Liu, S. Yuan, and L. Qiu, "Development of a PZT-based wireless digital monitor for composite impact monitoring," Smart Materials and Structures, vol. 21, no. 3, Article ID 035018, 2012.

[39] J. Wu, S. Yuan, X. Zhao, Y. Yin, and W. Ye, "A wireless sensor network node designed for exploring a structural health monitoring application," Smart Materials and Structures, vol. 16, no. 5, pp. 1898-1906, 2007.

[40] J. Wu, S. Yuan, Y. Shang, and Z. Wang, "Strain distribution monitoring wireless sensor network design and its evaluation research on aircraft wingbox," International Journal of Applied Electromagnetics and Mechanics, vol. 31, no. 1, pp. 17-28, 2009.

[41] T. Becker, M. Kluge, J. Schalk et al., "Autonomous sensor nodes for aircraft structural health monitoring," IEEE Sensors Journal, vol. 9, no. 11, pp. 1589-1595, 2009.

[42] S. W. Arms, C. P. Townsend, D. L. Churchill et al., "Energy harvesting, wireless, structural health monitoring and reporting system," in Proceedings of the 2nd Asia-Pacific Workshop on SHM, Melburn, Australia, 2008.

[43] S. W. Arms, J. H. Galbreath, C. P. Townsend et al., "Energy harvesting wireless sensors and networked timing synchronization for aircraft structural health monitoring," in Proceedings of the 1st International Conference on Wireless Communication, Vehicular Technology, Information Theory and Aerospace and Electronic Systems Technology (Wireless VITAE '09), pp. 16-20, Aalborg, Denmark, May 2009.

[44] S. W. Arms, C. P. Townsend, J. H. Galbreath, S. J. DiStasi, D. Liebschutz, and N. Phan, "Flight testing of wireless sensing networks for rotorcraft structural health and usage management systems," in Proceedings of the 14th Australian International Aerospace Congress, Melbourne, Australia, 2011.

[45] S. Gao, S. Yuan, L. Qiu, B. Ling, and Y. Ren, "A high-throughput multi-hop WSN for structural health monitoring," Journal of Vibroengineering, vol. 18, no. 2, pp. 781-800, 2016.
[46] S. Gao, X. Dai, Z. Liu, and G. Tian, "High-performance wireless piezoelectric sensor network for distributed structural health monitoring," International Journal of Distributed Sensor Networks, vol. 2016, Article ID 3846804, 16 pages, 2016.

[47] International Telecommunications Union, Final Acts World Radio Conference 15, vol. 1, International Telecommunications Union, 2015.

[48] D. W. Matolak and A. Chandrasekaran, "Aircraft intra-vehicular channel characterization in the $5 \mathrm{GHz}$ band," in Proceedings of the Integrated Communications, Navigation and Surveillance Conference (ICNS '08), pp. 1-6, IEEE, Bethesda, Md, USA, May 2008.

[49] J. Engelbrecht, T. Fuss, U. Schwark, and O. Michler, "Measurement of interference path loss between wireless avionics intracommunications system and aircraft systems at $4.2-4.4 \mathrm{GHz}$ band," in Proceedings of the 10th Loughborough Antennas and Propagation Conference (LAPC '14), pp. 119-123, November 2014.

[50] ITU, Technical Characteristics and Spectrum Requirements of Wireless Avionics Intra-Communications Systems to Support Their Safe Operation (ITU-R Report M.2283-0), International Telecommunications Union, 2013.

[51] N. Raharya and M. Suryanegara, "Compatibility analysis of Wireless Avionics Intra Communications (WAIC) to radio altimeter at $4200-4400 \mathrm{MHz}$," in Proceedings of the International Conference on IEEE Asia Pacific Conference on Wireless and Mobile (APWiMob '14), pp. 17-22, August 2014.

[52] D. Samson, M. Kluge, T. Becker, and U. Schmid, "Wireless sensor node powered by aircraft specific thermoelectric energy harvesting," Sensors and Actuators A: Physical, vol. 172, no. 1, pp. 240-244, 2011.

[53] Y. Lu, A. Savvaris, A. Tsourdos, and M. Bevilacqua, "Vibration energy harvesters for wireless sensor networks for aircraft health monitoring," in Proceedings of the IEEE Metrology for Aerospace, pp. 25-32, Florence, Italy, June 2016.

[54] G. A. Hart, S. D. Moss, D. J. Nagle et al., "Vibration energy harvesting for aircraft, trains and boats," in Proceeding of the Acoustics, pp. 17-20, Victor Harbor, Australia, 2013.

[55] Z. Hadas, V. Vetiska, R. Huzlik, and V. Singule, "Modelbased design and test of vibration energy harvester for aircraft application," Microsystem Technologies, vol. 20, no. 4-5, pp. 831$843,2014$.

[56] N. Siu, N. Titchener, A. Uranga, and E. Greitzer, "Evaluating propulsor mechanical flow power in powered aircraft wind tunnel experiments," AIAA Journal, vol. 19, pp. 2350-2371, 2013.

[57] A. T. Klesh and P. T. Kabamba, "Solar-powered aircraft: energyoptimal path planning and perpetual endurance," Journal of Guidance Control \& Dynamics, vol. 32, no. 4, pp. 1320-1329, 2015.

[58] J. A. R. Azevedo and F. E. S. Santos, "Energy harvesting from wind and water for autonomous wireless sensor nodes," IET Circuits, Devices \& Systems, vol. 6, no. 6, pp. 413-420, 2012.

[59] W. Zhao, K. Choi, Z. Dilli, S. Bauman, T. Salter, and M. Peckerar, "Design of radio frequency energy harvesting system for an unmanned airplane," in Proceedings of the International Semiconductor Device Research Symposium (ISDRS '11), pp. 1-2, College Park, Md, USA, December 2011.

[60] J. A. Hall Jr., S. M. Loo, D. Stephenson et al., "A portable wireless particulate sensor system for continuous real-time environmental monitoring," in Proceedings of the 42nd International Conference on Environmental Systems, San Diego, Calif, USA, July 2012. 
[61] J. A. Hall Jr., J. Kiepert, M. Pook, and S. M. Loo, "Monitoring of aircraft cabin particulate matter concentrations using a wireless sensor network," in Proceedings of the 43rd International Conference on Environmental Systems (ICES '13), pp. 1-7, Vail, Colo, USA, July 2013.

[62] S. F. Yuan, Z. L. Wang, L. Qiu, Y. Wang, and M. L. Liu, "A multiradio sink node designed for wireless SHM applications," Smart Structures and Systems, vol. 11, no. 3, pp. 261-282, 2013.

[63] J. Wu, S. Yuan, G. Zhou, S. Ji, Z. Wang, and Y. Wang, "Design and evaluation of a wireless sensor network based aircraft strength testing system," Sensors, vol. 9, no. 6, pp. 4195-4210, 2009.

[64] C. Delebarre, S. Grondel, and F. Rivart, "Autonomous piezoelectric structural health monitoring system for on-production line use," Advances in Applied Ceramics, vol. 114, no. 4, pp. 205-210, 2015.

[65] S. M. Loo, M. Owen, J. Kiepert, and A. Planting, "Modular, portable, reconfigurable, and wireless sensing system for the aircraft cabin," Journal of ASTM International, vol. 5, no. 4, pp. $1-9,2008$.

[66] X. Zhao, H. Gao, G. Zhang et al., "Active health monitoring of an aircraft wing with embedded piezoelectric sensor/actuator network: I. Defect detection, localization and growth monitoring," Smart Materials and Structures, vol. 16, no. 4, article 032, pp. 1208-1217, 2007.

[67] X. Zhao, T. Qian, G. Mei et al., "Active health monitoring of an aircraft wing with an embedded piezoelectric sensor/actuator network: II. Wireless approaches," Smart Materials and Structures, vol. 16, no. 4, article 033, pp. 1218-1225, 2007.

[68] J. Blanckenstein, J. Garcia-Jimenez, J. Klaue, and H. Karl, "A scalable redundant TDMA protocol for high-density WSNs inside an aircraft," in Real-World Wireless Sensor Networks, vol. 281 of Lecture Notes in Electrical Engineering, pp. 165-177, Springer, 2014.

[69] D. Krichen, W. Abdallah, and N. Boudriga, "On the design of an embedded wireless sensor network for aircraft vibration monitoring using efficient game theoretic based MAC protocol," Ad Hoc Networks, vol. 61, pp. 1-15, 2017.

[70] D. Krichen, W. Abdallah, and N. Boudriga, "WSN-based flutter control application for aircraft wings structural health monitoring," in Proceedings of the 29th Annual ACM Symposium on Applied Computing, pp. 680-685, Gyeongju, Republic of Korea, March 2014.

[71] S. Chowdhury, S. Dutta, K. Mitra, D. K. Sanyal, M. Chattopadhyay, and S. Chattopadhyay, "Game-theoretic modeling and optimization of contention-prone medium access phase," in Proceedings of the 3rd International Conference on Broadband Communications, Information Technology \& Biomedical Applications, pp. 335-342, Gauteng, South Africa, November 2008.

[72] M. Barcelo, J. L. Vicario, G. Seco-Granados, J. M. Puig, and J. M. Laborda, "Multi-channel routing algorithm for clustertree wireless sensor networks in aerospace applications," in Proceedings of the 4th Annual Caneus Fly by Wireless Workshop, pp. 1-4, Montreal, Canada, June 2011.

[73] J. Ma, J. Peng, G. Zhang, Y. Zeng, X. Liu, and H. Meng, "Wireless sensor network system for aircraft condition monitoring," Measurement \& Control Technology, vol. 28, no. 11, pp. 6-13, 2009.

[74] H. Guanglin and X. Hongyu, "Design and simulation of the vibration monitoring system based on wireless sensor network," in Proceedings of the International Conference on Information Management, Innovation Management and Industrial Engineering, pp. 12-15, Sanya, China, October 2012.
[75] J. K. Notay and G. A. Safdar, "A wireless sensor network based structural health monitoring system for an airplane," in Proceedings of the 17th International Conference on Automation and Computing (ICAC '11), pp. 240-245, Huddersfield, UK, September 2011.

[76] G. Park, T. Rosing, M. D. Todd, C. R. Farrar, and W. Hodgkiss, "Energy harvesting for structural health monitoring sensor networks," Journal of Infrastructure Systems, vol. 14, no. 1, pp. 64-79, 2008.

[77] W. Zhou and B. Jing, "A study on the wireless sensor networks MAC protocol for aircraft health monitoring," vol. 295 of Lecture Notes in Electrical Engineering, pp. 131-143, Springer, Berlin, Germany, 2014.

[78] G. S. Rao and V. Vallikumari, "A beneficial analysis of node deployment schemes for wireless sensor networks," International Journal of Advanced Smart Sensor Network Systems, vol. 2, no. 2, 2012.

[79] D. J. Cichon and T. Kürner, "Propagation prediction models," COST 231 Final Report, COST Telecom Secretariat, Brussels, Belgium, 1999.

[80] J. Naganawa, H. Miyazaki, and H. Tajima, "Measurement-based evaluation on detection probability of extended squitter for air-to-ground surveillance," IEEE Transactions on Vehicular Technology, vol. 66, no. 10, pp. 8883-8894, 2017.

[81] A. Motley and J. Keenan, "Radio coverage in buildings," British Telecom Technical Journal, vol. 8, no. 1, pp. 19-24, 1990.

[82] A. M. D. Turkmani, "Modelling of radio transmissions into and within multistory buildings at 900, 1800 and $2300 \mathrm{MHz}$," IEEE Proceddings-I, vol. 140, pp. 462-470, 1993.

[83] P. Jacob, R. P. Sirigina, A. S. Madhukumar, and V. A. Prasad, "Cognitive radio for aeronautical communications: a survey," IEEE Access, vol. 4, pp. 3417-3443, 2016.

[84] N. L. Armstrong and Y. M. M. Antar, "Investigation of the electromagnetic interference threat posed by a wireless network inside a passenger aircraft," IEEE Transactions on Electromagnetic Compatibility, vol. 50, no. 2, pp. 277-284, 2008.

[85] M. Bocca, L. M. Eriksson, A. Mahmood, R. Jäntti, and J. Kullaa, "A synchronized wireless sensor network for experimental modal analysis in structural health monitoring," ComputerAided Civil and Infrastructure Engineering, vol. 26, no. 7, pp. 483-499, 2011.

[86] M. Ceriotti, L. Mottola, G. P. Picco et al., "Monitoring heritage buildings with wireless sensor networks: the Torre Aquila deployment," in Proceedings of the 8th ACM/IEEE International Conference, pp. 277-288, San Francisco, Calif, USA, April 2009.

[87] E. G. Straser and A. S. Kiremidjian, "A modular, wireless damage monitoring system for structures," Tech. Rep. 128, Department of Civil and Environmental Engineering, Stanford University, Stanford, Calif, USA, 1998.

[88] S. Ganeriwal, R. Kumar, and M. B. Srivastava, "Timing-sync protocol for sensor networks," in Proceedings of the 1st International Conference on Embedded Networked Sensor Systems (SenSys '03), pp. 138-149, ACM, Los Angeles, Calif, USA, November 2003.

[89] J. Elson, L. Girod, and D. Estrin, "Fine-grained network time synchronization using reference broadcasts," ACM SIGOPS Operating Systems Review, vol. 36, pp. 147-163, 2002.

[90] M. Maróti, B. Kusy, G. Simon, and Á. Lédeczi, “The flooding time synchronization protocol," in Proceedings of the 2nd International Conference on Embedded Networked Sensor Systems, ACM, 2004. 
[91] S. Kim, S. Pakzad, D. Culler, J. Demmel, and G. Fenves, "Health monitoring of civil infrastructures using wireless sensor networks," in Proceedings of IEEE International Symposium on Information Processing in Sensor Networks, pp. 254-263, 2007.

[92] F. Osterlind and A. Dunkels, "Approaching the maximum 802.15.4 multi-hop throughput," in Proceedings of the 5th ACM Workshop on Embedded Networked Sensors, pp. 432-456, 2008.

[93] D. Vassis, G. Kormentzas, and C. Skianis, "Performance evaluation of single and multi-channel actor to actor communication for wireless sensor actor networks," Ad Hoc Networks, vol. 4, no. 4, pp. 487-498, 2006.

[94] C. E.-A. Campbell, S. Khan, D. Singh, and K.-K. Loo, "Multichannel multi-radio using 802.11 based media access for sink nodes in wireless sensor networks," Sensors, vol. 11, no. 5, pp. 4917-4942, 2011.

[95] X. Wei, Q. Wang, T. Wang, and J. Fan, "Jammer localization in multi-hop wireless network: a comprehensive survey," IEEE Communications Surveys \& Tutorials, vol. 19, no. 2, pp. 765-799, 2017.

[96] S. H. Choi, D. H. Hwang, and Y. H. Choi, "Wireless intrusion prevention system using dynamic random forest against wireless MAC spoofing attack," in Proceedings of the IEEE Conference on Dependable and Secure Computing, pp. 131-137, Taipei, Taiwan, August 2017.

[97] V. Kumar, S. Chakraborty, F. A. Barbhuiya, and S. Nandi, "Detection of stealth Man-in-the-Middle attack in wireless LAN," in Proceedings of the 2nd IEEE International Conference on Parallel, Distributed and Grid Computing, pp. 290-295, December 2012.

[98] Y. Mo, E. Garone, A. Casavola et al., "False data injection attacks against state estimation in wireless sensor networks," in Proceedings of the IEEE Decision and Control Conference, pp. 5967-5972, 2011.

[99] F. A. El-Moussa, N. Linge, and M. Hope, "Active router approach to defeating denial-of-service attacks in networks," IET Communications, vol. 1, no. 1, pp. 55-63, 2007.

[100] C. L. Schuba, I. V. Krsul, M. G. Kuhn, E. H. Spafford, A. Sundaram, and D. Zamboni, "Analysis of a denial of service attack on TCP," in Proceedings of the IEEE Symposium on Security and Privacy, pp. 208-223, May 1997.

[101] R. K. C. Chang, "Defending against flooding-based distributed denial-of-service attacks: a tutorial," IEEE Communications Magazine, vol. 40, no. 10, pp. 42-51, 2002.

[102] A. Kieyzun, P. J. Guo, K. Jayaraman, and M. D. Ernst, "Automatic creation of SQL Injection and cross-site scripting attacks," in Proceedings of the IEEE 31st International Conference on Software Engineering, pp. 199-209, Vancouver, Canada, May 2009.

[103] A. Moosa, "Artificial neural network based web application firewall for SQL injection," World Academy of Science, Engineering and Technology, vol. 64, pp. 12-21, 2010.

[104] https://zh.scribd.com/document/37108247/Micro-Strain-Wireless-Sensors-Aircraft-Structural-Health-Monitoring-2008.

[105] O. Elmazria and T. Aubert, "Wireless SAW sensor for high temperature applications: material point of view," in Proceedings of the 14th Australian International Aerospace Congress, p. 10, Melbourne, Australia, 2011.

[106] W. C. Wilson and G. M. Atkinson, "Wireless sensor applications in extreme aeronautical environments," in Proceedings of the IEEE International Conference on Wireless for Space and Extreme Environments (WiSEE '13), pp. 1-6, Baltimore, Md, USA, November 2013.
[107] S. Yuan, Y. Ren, L. Qiu, and H. Mei, "A multi-response-based wireless impact monitoring network for aircraft composite structures," IEEE Transactions on Industrial Electronics, vol. 63, no. 12, pp. 7712-7722, 2016.

[108] I. Demirkol, C. Ersoy, and F. Alagöz, "MAC protocols for wireless sensor networks: a survey," IEEE Communications Magazine, vol. 44, no. 4, pp. 115-121, 2006.

[109] J. Liu, I. Demirkiran, T. Yang, and A. Helfrick, "Feasibility study of IEEE 802.15.4 for aerospace wireless sensor networks," in Proceedings of the IEEE/AIAA 28th Digital Avionics Systems Conference, Orlando, Fla, USA, October 2009.

[110] A. Mishra, F. Gondal, A. Afrashteh, and R. Wilson, "Embedded wireless sensors for aircraft/automobile tire structural health monitoring," in Proceedings of the 2nd IEEE Workshop on Wireless Mesh Networks, pp. 163-165, Reston, Va, USA, September 2006.

[111] R. S. Wagner, "Standards-based wireless sensor networking protocols for spaceflight applications," in Proceedings of the IEEE Aerospace Conference, pp. 1-7, Big Sky, Mont, USA, March 2010.

[112] K. Akkarajitsakul, E. Hossain, D. Niyato, and D. I. Kim, "Game theoretic approaches for multiple access in wireless networks: a survey," IEEE Communications Surveys \& Tutorials, vol. 13, no. 3, pp. 372-395, 2011.

[113] P. E. Omiyi, K. Bür, and Y. Yang, "Distributed convergecast scheduling for reduced interference in wireless sensor and actuator networks," in Proceedings of the IEEE Wireless Communications and Networking Conference, pp. 1-5, Sydney, Australia, April 2010.

[114] X. Dai, P. E. Omiyi, K. Bür, and Y. Yang, "Interference-aware convergecast scheduling in wireless sensor/actuator networks for active airflow control applications," Wireless Communications and Mobile Computing, vol. 14, no. 3, pp. 396-408, 2014.

[115] W. Dargie, "Dynamic power management in wireless sensor networks: State-of-the-art," IEEE Sensors Journal, vol. 12, no. 5, pp. 1518-1528, 2012.

[116] T. Torfs, T. Sterken, S. Brebels et al., "Low power wireless sensor network for building monitoring," IEEE Sensors Journal, vol. 13, no. 3, pp. 909-915, 2013.

[117] D. Vujic, R. Stojić, and Z. Filipović, "Wireless sensor networks technology in aircraft structural health monitoring," in Proceedings of the 5th International Conference on Defensive Technologies (OTEH '12), pp. 141-147, Belgrade, Serbia, 2012.

[118] D. Vujic, "Wireless sensor networks applications in aircraft structural health monitoring," Journal of Applied Engineering Science, vol. 13, no. 2, pp. 79-86, 2015.

[119] K. Akkaya and M. Younis, "A survey on routing protocols for wireless sensor networks," Ad Hoc Networks, vol. 3, no. 3, pp. 325-349, 2005.

[120] X. U. Jingjing, B. Chen, and H. Qian, "A WSN node deployment algorithm for airport noise monitoring," Electronic Science \& Technology, vol. 7, pp. 14-18, 2014.

[121] The Internet of Things, "Cisco visualizations," https://www .cisco.com/go/iot.

[122] FP7 project IoT6 "Researching IPv6 potential for the Internet", http://iot6.eu/.

[123] Internet of Things, "Architecture, FP7 project," https://www.iso .org/standard/65695.html.

[124] ISO/IEC 30141, Internet of Things Reference Architecture (IoT RA). 


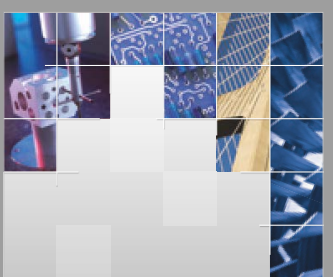

\section{Enfincering}
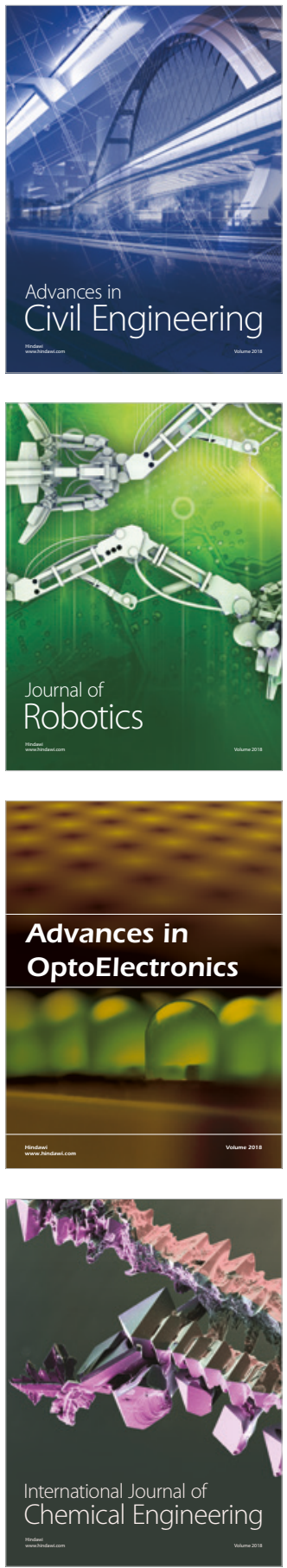

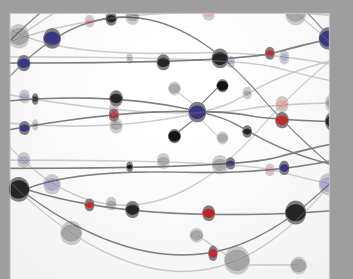

\section{Rotating \\ Machinery}

The Scientific World Journal

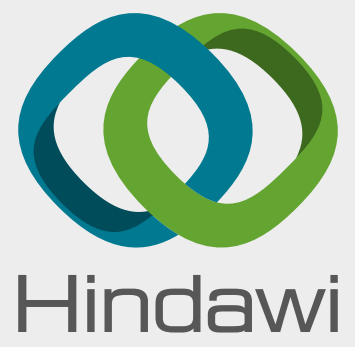

Submit your manuscripts at

www.hindawi.com
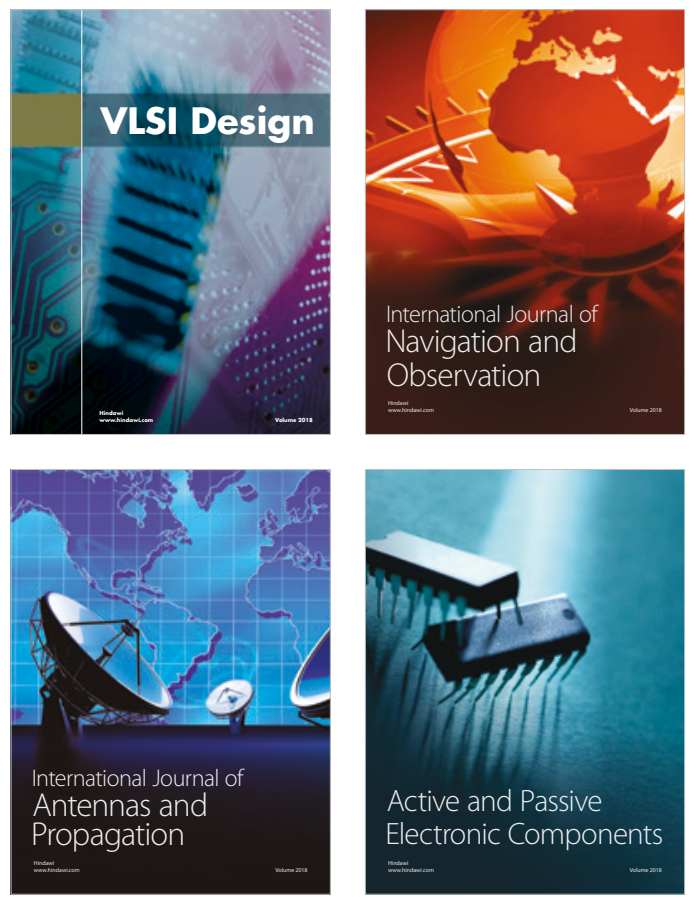
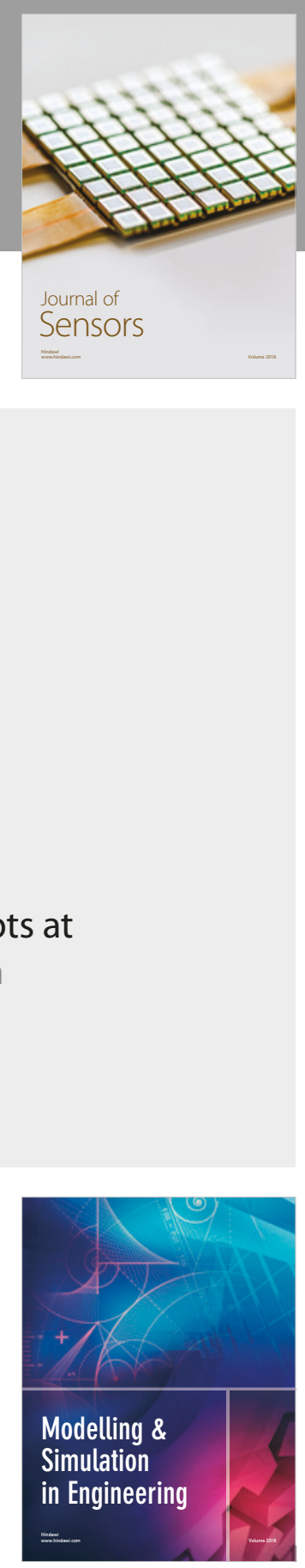

\section{Advances \\ Multimedia}
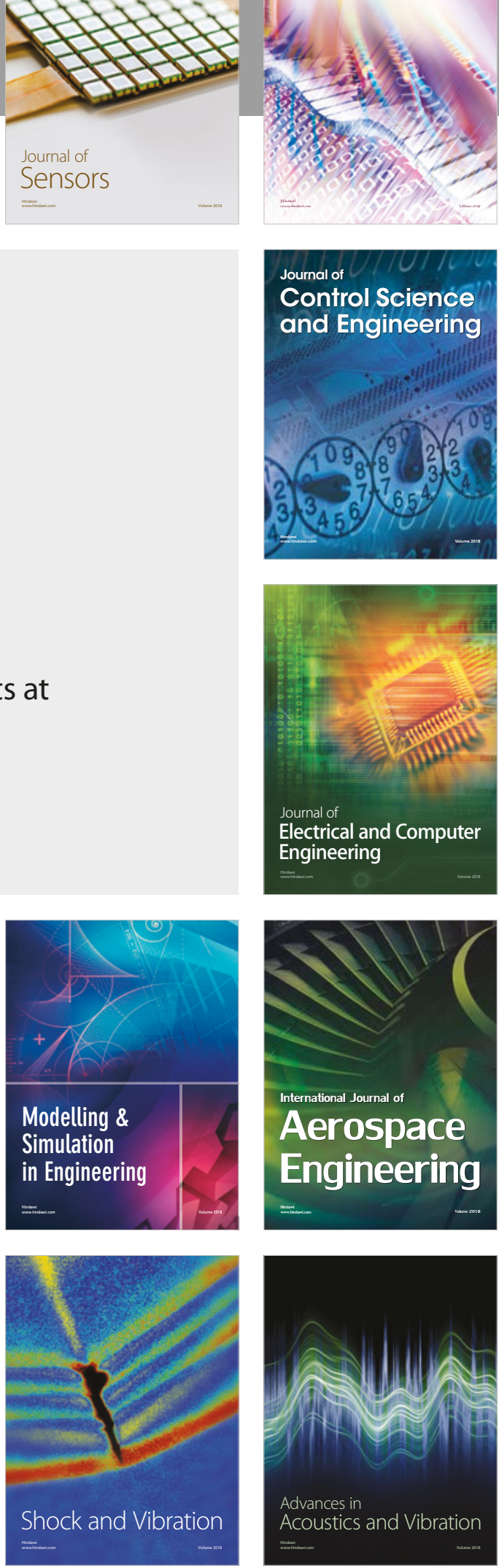